\title{
The Mu2e Experiment
}

\section{Tomo Miyashita}

Caltech

\section{On Behalf of the Mu2e Collaboration}

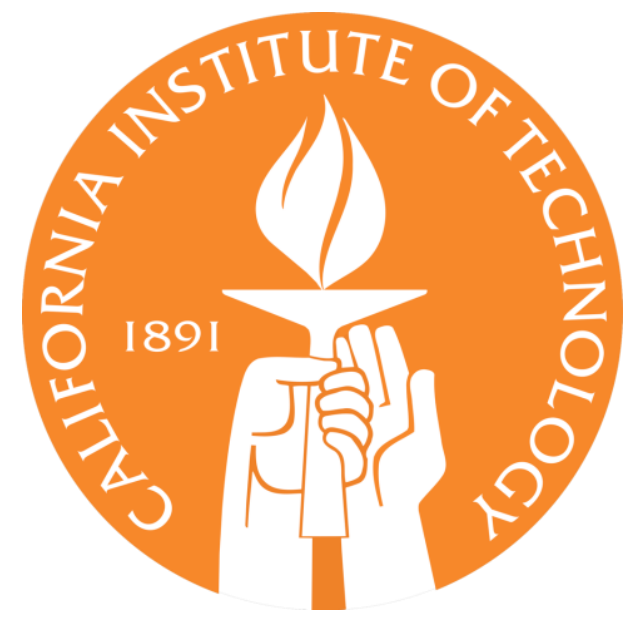

\section{CIPANP 2018}

Palm Springs, CA

May 29th, 2018

This document was prepared by Mu2e collaboration using the resources of the Fermi National Accelerator Laborator (Fermilab), a U.S. Department of Energy, Office of Science, HEP User Facility. Fermilab is managed by Ferm Research Alliance, LLC (FRA), acting under Contract No. DE-AC02-07CH11359.

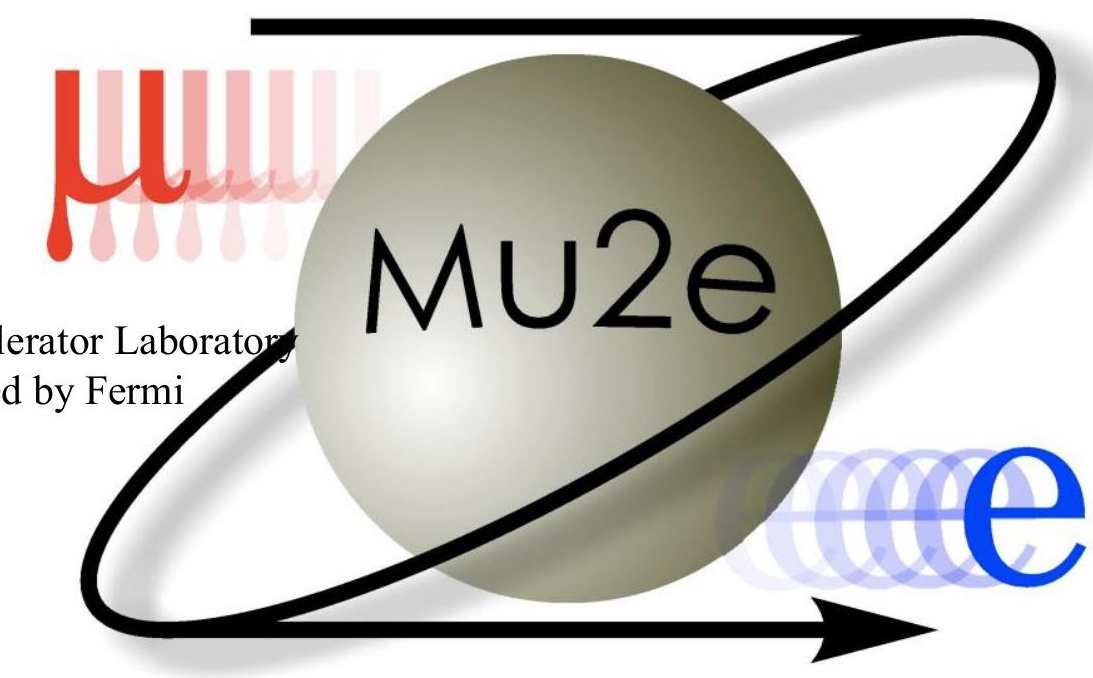




\section{Overview}

- Motivation and Theory

- Experiment Overview

- Experiment Design

- Proton Beam

- Production and Stopping Targets

- Tracker

- Calorimeter

- $\mathrm{CRV}$

- $\mathrm{DAQ} /$ Trigger

- Mu2e Schedule

- Mu2e II

- Summary 


\section{Motivation}

- Mu2e is searching for Charged Lepton Flavor Violation (CLFV)

- Specifically, the conversion of a $\mu^{-}$to an $e^{-}$in the field of a nucleus:

$$
\mu^{-}+A(Z, N) \rightarrow e^{-}+A(Z, N)
$$

- Using the current Fermilab accelerator complex, we intend to achieve a sensitivity 4 orders of magnitude better than current limits:

$$
\begin{gathered}
\text { Target Sensitivity: } \\
R_{\mu e}=\frac{\Gamma\left[\mu^{-}+A(Z, N) \rightarrow e^{-}+A(Z, N)\right]}{\Gamma\left[\mu^{-}+A(Z, N) \rightarrow \nu_{\mu}+A(Z-1, N+1)\right]}<6.7 \times 10^{-17}(90 \% \mathrm{CL}) \\
4 \text { orders of magnitude better than current limits: } \\
\text { [INDRUM Bertl et al, Eur. Phys. J. C 47, 337-346 (2006)] }
\end{gathered}
$$

- We will have discovery sensitivity over a broad range of New Physics parameter space 


\section{Flavor Violation}

- We have known for a long time that quarks mix $\Rightarrow$ (Quark) Flavor Violation

- Mixing strengths parameterized by the CKM matrix

- We have known since 2001 that neutrinos can also mix $\Rightarrow$ (Neutral) Lepton Flavor Violation

- Mixing strengths parameterized by the PMNS matrix

- Why not charged leptons as well?

- Charged Lepton Flavor Violation (CLFV) 


\section{CLFV in the Standard Model}

- CLFV is not technically allowed in the SM because since charged lepton number is accidentally conserved when neutrinos are massless

- However, if we include massive neutrinos in our model then CLFV becomes possible at the loop level due to neutrino oscillations:

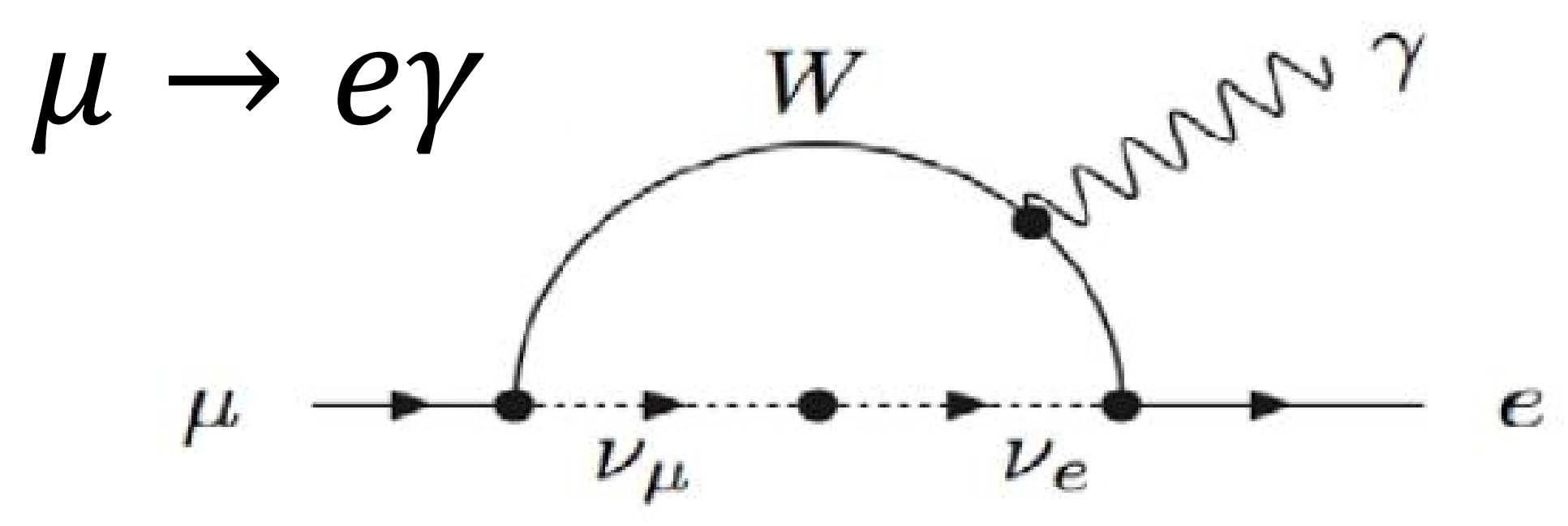

- This process is extremely suppressed $\left(\mathcal{B}\left(\mu^{-} \rightarrow e^{-} \gamma\right)<10^{-54}\right)$

- Therefore, any signal at our sensitivity would be a sign of new physics 


\section{Example CLFV Processes}

- Potential channels for CLFV searches:

\begin{tabular}{l|l|l}
\hline \multicolumn{1}{c|}{ Process } & \multicolumn{1}{c}{ Current Limit } & Next Generation exp \\
\hline$\tau \rightarrow \mu \eta$ & $\mathrm{BR}<6.5 \times 10^{-8}$ & \\
$\tau \rightarrow \mu \gamma$ & $\mathrm{BR}<6.8 \times 10^{-8}$ & $10^{-9}-10^{-10}$ (Belle II) \\
$\tau \rightarrow \mu \mu \mu$ & $\mathrm{BR}<3.2 \times 10^{-8}$ & \\
$\tau \rightarrow e e e$ & $\mathrm{BR}<3.6 \times 10^{-8}$ & \\
\hline$K_{\mathrm{L} \rightarrow e \mu}$ & $\mathrm{BR}<4.7 \times 10^{-12}$ & \\
$K^{+} \rightarrow \pi^{+} e^{-} \mu^{+}$ & $\mathrm{BR}<1.3 \times 10^{-11}$ & \\
\hline$B^{0} \rightarrow e \mu$ & $\mathrm{BR}<7.8 \times 10^{-8}$ & \\
$B^{+} \rightarrow K^{+} e \mu$ & $\mathrm{BR}<9.1 \times 10^{-8}$ & $10^{-14}$ (MEG Upgrade) \\
\hline$\mu^{+} \rightarrow e^{+} \gamma$ & $\mathrm{BR}<4.2 \times \times 10^{-13}$ & $10^{-16}$ (Mu3e) \\
$\mu^{+} \rightarrow e^{+} e^{+} e^{-}$ & $\mathrm{BR}<1.0 \times 10^{-12}$ & $10^{-17}$ (Mu2e, COMET) \\
$\mu N \rightarrow e N$ & $\mathrm{R}{ }_{\mu \mathrm{e}}<7.0 \times 10^{-13}$ & \\
\hline
\end{tabular}

- Although CLFV $\tau$ processes could have larger branching ratios than $\mu$ processes, dedicated muon experiments can produces $\mathrm{O}\left(10^{10}\right) \mu / \mathrm{s}$ whereas colliders produce $\mathrm{O}\left(10^{10}\right) \tau /$ year 


\section{New Physics Reach}

- There are many possible new physics contributions to $\mu \mathrm{N} \rightarrow e \mathrm{~N}$, either through loops or the exchange of heavy intermediate particles

- Many NP models predict rates observable at next gen CLFV experiments

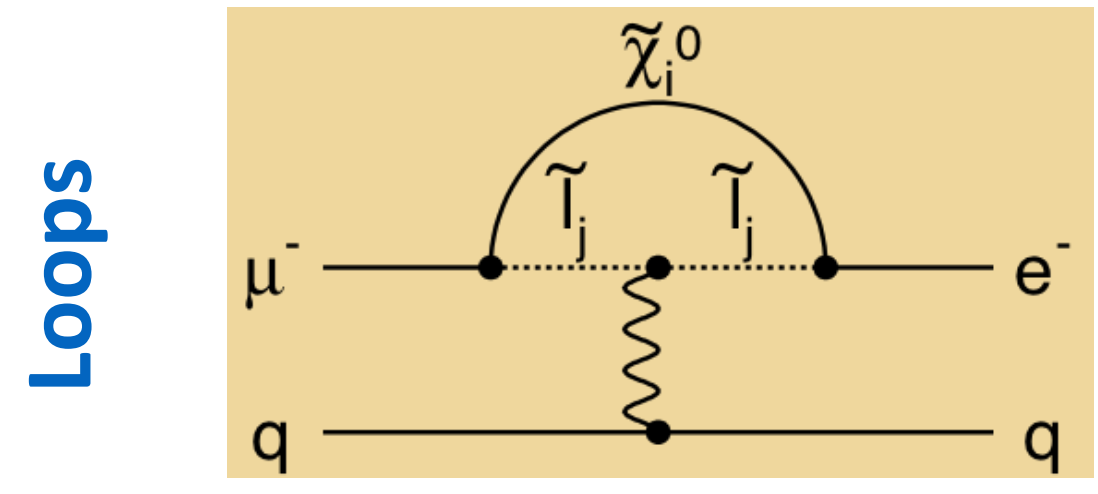

Supersymmetry

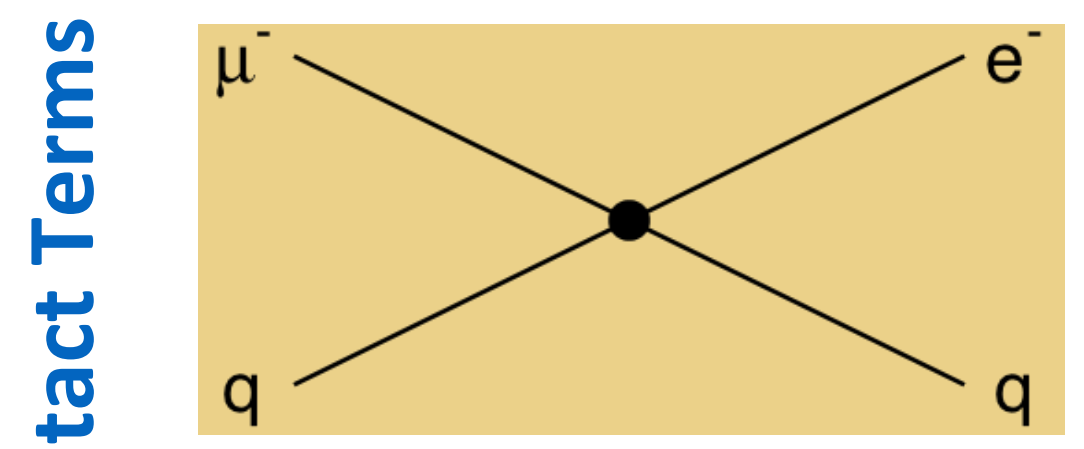

Compositeness

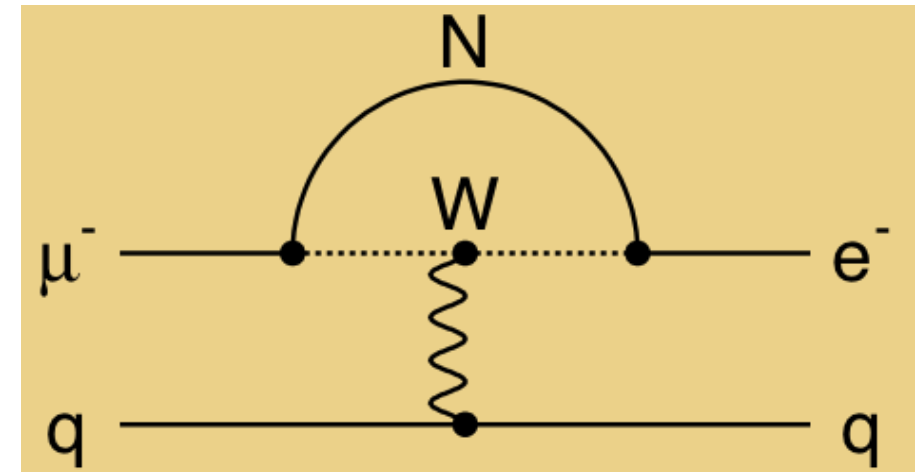

Heavy Neutrinos

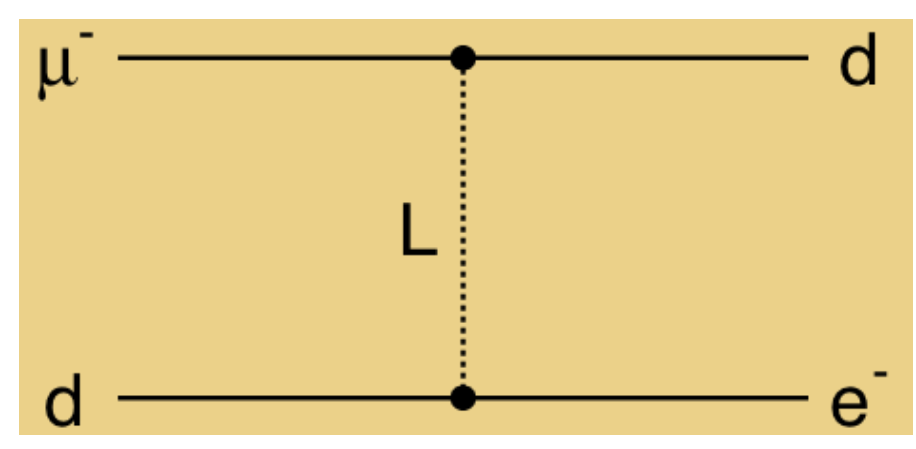

Leptoquarks

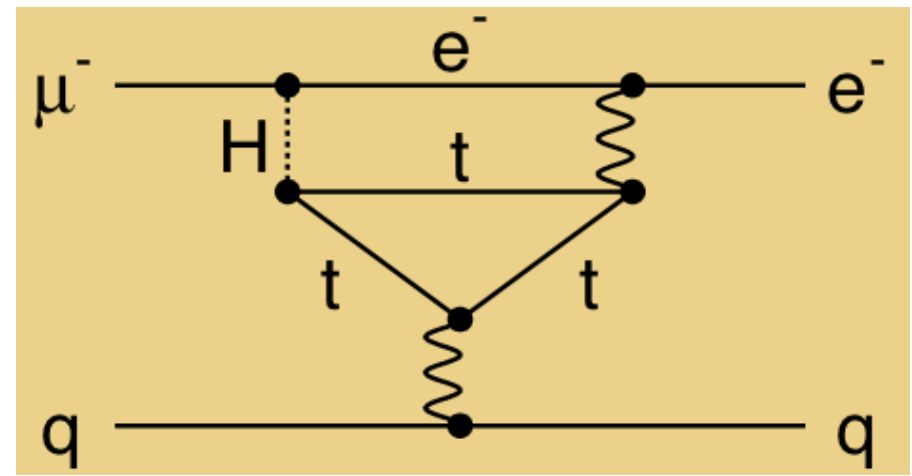

Two Higgs Doublets

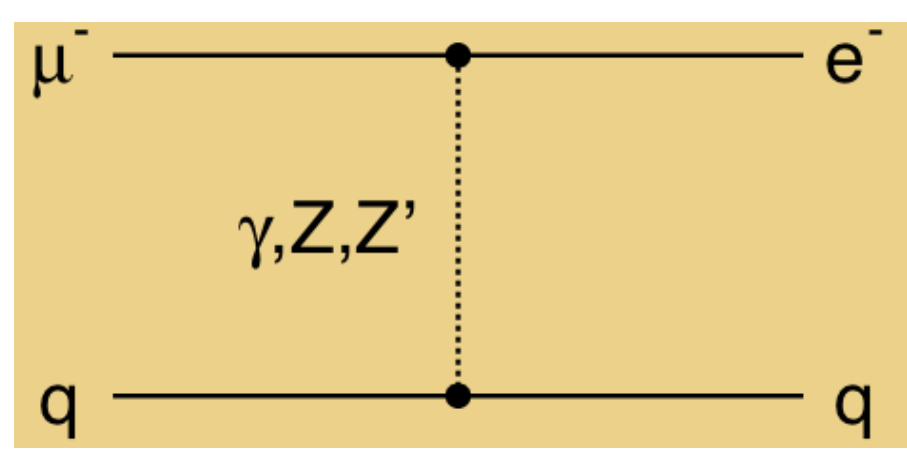

New Heavy Bosons / Anomalous Couplings 


\section{Model-Independent Effective Lagrangian}

$$
L_{\mathrm{CLFV}}=\frac{m_{m}}{(1+k) \Lambda^{2}} \bar{m}_{R} s_{m n} e_{L} F^{m n}+\frac{k}{(1+k) \Lambda^{2}} \bar{m}_{L} g_{m} e_{L}\left(\bar{u}_{L} g_{m} u_{L}+\bar{d}_{L} g_{m} d_{L}\right)+\text { h.c. }
$$

$\Lambda$ : effective mass scale of New Physics
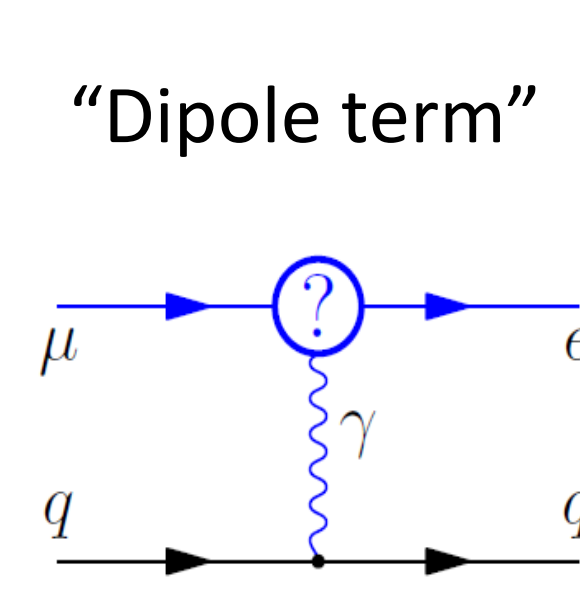

Contributes to

$$
\mu \rightarrow e \gamma
$$

- $\quad$ CLFV can probe very high mass scales $\mathrm{O}(1000-10,000$ $\mathrm{TeV})$

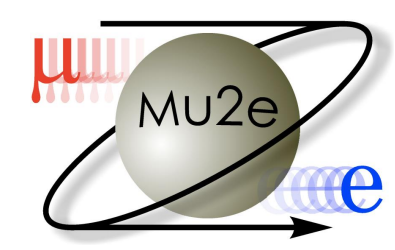

$k$ : relative contribution of the contact term

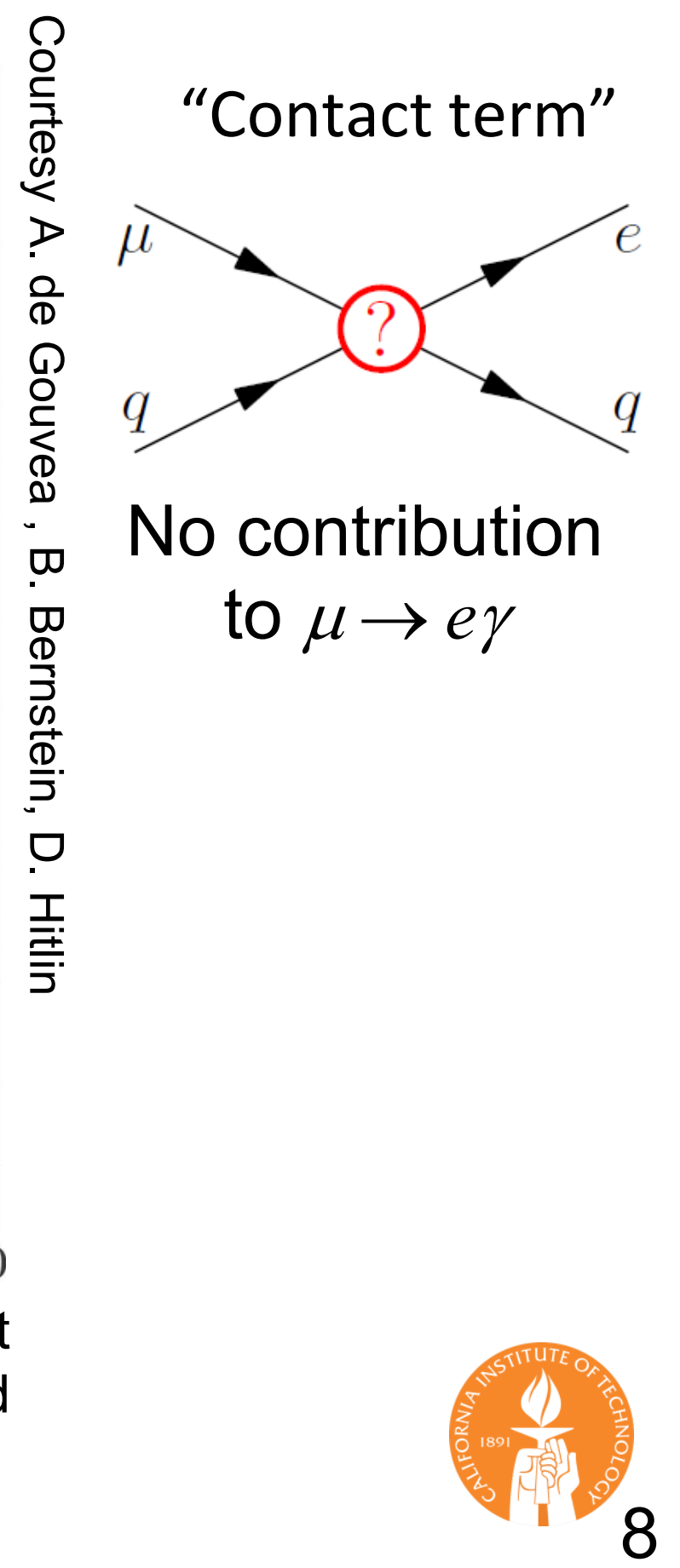




\section{Motivation II}

- Mu2e has discovery sensitivity across a wide range of models:

W. Altmannshofer, A.J.Buras, S.Gori, P.Paradisi, D.M.Straub

\begin{tabular}{|c|c|c|c|c|c|c|c|}
\hline & $\mathrm{AC}$ & RVV2 & $\mathrm{AKM}$ & $\delta \mathrm{LL}$ & FBMSSM & LHT & RS \\
\hline$D^{0}-\bar{D}^{0}$ & $\star \star \star \star$ & $\star$ & $\star$ & $\star$ & $\star$ & $\star \star \star$ & $?$ \\
\hline$\epsilon_{K}$ & $\star$ & $\star \star \star$ & $\star \star \star$ & $\star$ & $\star$ & $\star \star$ & $\star \star \star \star$ \\
\hline$S_{\psi \phi}$ & $\star \star \star$ & $\star \star \star$ & $\star \star \star$ & $\star$ & $\star$ & $\star \star \star$ & $\star \star \star$ \\
\hline$S_{\phi K_{S}}$ & $\star \star \star$ & $\star \star$ & $\star$ & $\star \star \star$ & $\star \star \star$ & $\star$ & $?$ \\
\hline$A_{\mathrm{CP}}\left(B \rightarrow X_{s} \gamma\right)$ & $\star$ & $\star$ & $\star$ & $\star \star \star$ & $\star \star \star$ & $\star$ & $?$ \\
\hline$A_{7,8}\left(B \rightarrow K^{*} \mu^{+} \mu^{-}\right)$ & $\star$ & $\star$ & $\star$ & $\star \star \star$ & $\star \star \star$ & $\star \star$ & $?$ \\
\hline$A_{9}\left(B \rightarrow K^{*} \mu^{+} \mu^{-}\right)$ & $\star$ & $\star$ & $\star$ & $\star$ & $\star$ & $\star$ & $?$ \\
\hline$B \rightarrow K^{(*)} \nu \bar{\nu}$ & $\star$ & $\star$ & $\star$ & $\star$ & $\star$ & $\star$ & $\star$ \\
\hline$B_{s} \rightarrow \mu^{+} \mu^{-}$ & $\star \star \star$ & $\star \star \star$ & $\star \star \star$ & $\star \star \star$ & $\star \star \star$ & $\star$ & $\star$ \\
\hline$K^{+} \rightarrow \pi^{+} \nu \bar{\nu}$ & $\star$ & $\star$ & $\star$ & $\star$ & $\star$ & $\star \star \star$ & $\star \star \star$ \\
\hline$K_{L} \rightarrow \pi^{0} \nu \bar{\nu}$ & $\star$ & $\star$ & $\star$ & $\star$ & $\star$ & $\star \star \star \star$ & $\star \star \star \star$ \\
\hline$\mu \rightarrow e \gamma$ & $\star \star \star$ & $\star \star \star$ & $\star \star \star$ & $\star \star \star$ & $\star \star \star$ & $\star \star \star$ & $\star \star \star$ \\
\hline$\tau \rightarrow \mu \gamma$ & 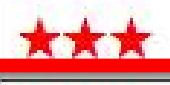 & $\star \star \star \star$ & $\star$ & 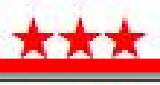 & $\star \star \star \star$ & $\star \star \star \star$ & $\star \star \star \star$ \\
\hline$\mu+N \rightarrow e+N$ & $\star \star \star$ & $\star \star \star$ & $\star \star \star$ & $\star \star \star$ & $\star \star \star$ & $\star \star \star$ & $\star \star \star$ \\
\hline$d_{n}$ & $\star \star \star \star$ & $\star \star \star \star$ & $\star \star \star \star$ & $\star \star$ & $\star \star \star \star$ & $\star$ & $\star \star \star$ \\
\hline$d_{e}$ & $\star \star \star$ & $\star \star \star$ & $\star \star$ & $\star$ & $\star \star \star$ & $\star$ & $\star \star \star$ \\
\hline$(g-2)_{\mu}$ & $\star \star \star$ & $\star \star \star$ & $\star \star$ & $\star \star \star$ & $\star \star \star$ & $\star$ & $?$ \\
\hline
\end{tabular}

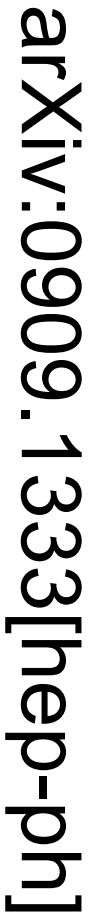

Table 8: "DNA" of flavour physics effects for the most interesting observables in a selection of SUSY and non-SUSY models $\star \star \star$ signals large effects, $\star \star$ visible but small effects and $\star$ implies that the given model does not predict sizable effects in that observable. 


\section{Experimental Concept}

- Generate a beam of low momentum muons

- Muons are stopped in an aluminum target

- When stopped muons convert to electrons, the nucleus recoils and the electron is emitted at a specific energy

- Signal is mono-energetic electron at $104.9 \mathrm{MeV}$

- Main background is Decay In Orbit (DIO) events

- To achieve our target sensitivity, we need $\sim 10^{18}$ stopped muons over 3 year run

$=>\sim 10^{10}$ stopped muons per second

\section{Coherent Conversion}

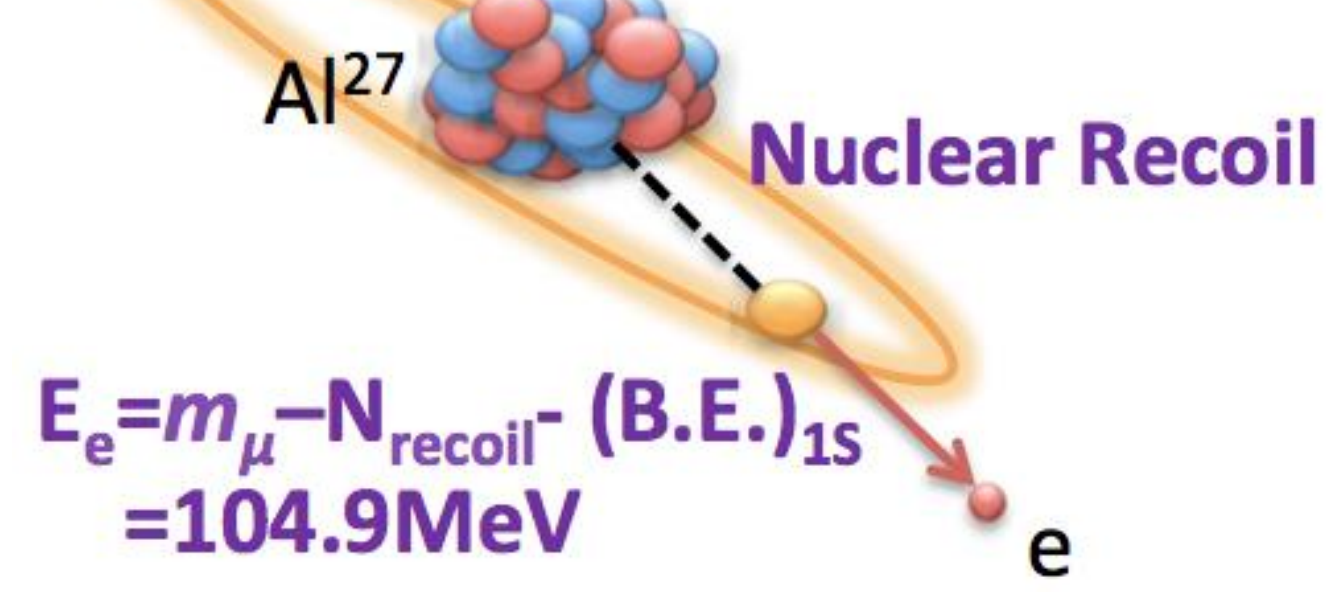

\section{Decay In Orbit}

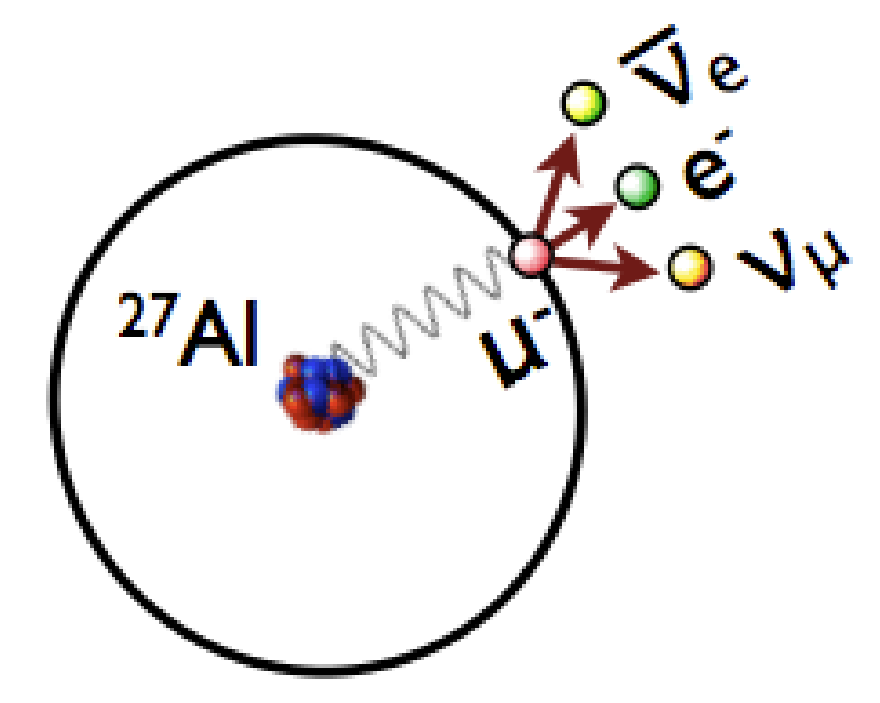




\section{Decay In Orbit Energy Distribution}

- Although naively, the maximum electron energy from muon decay should be far below our signal energy $(104.9 \mathrm{MeV}) \ldots$

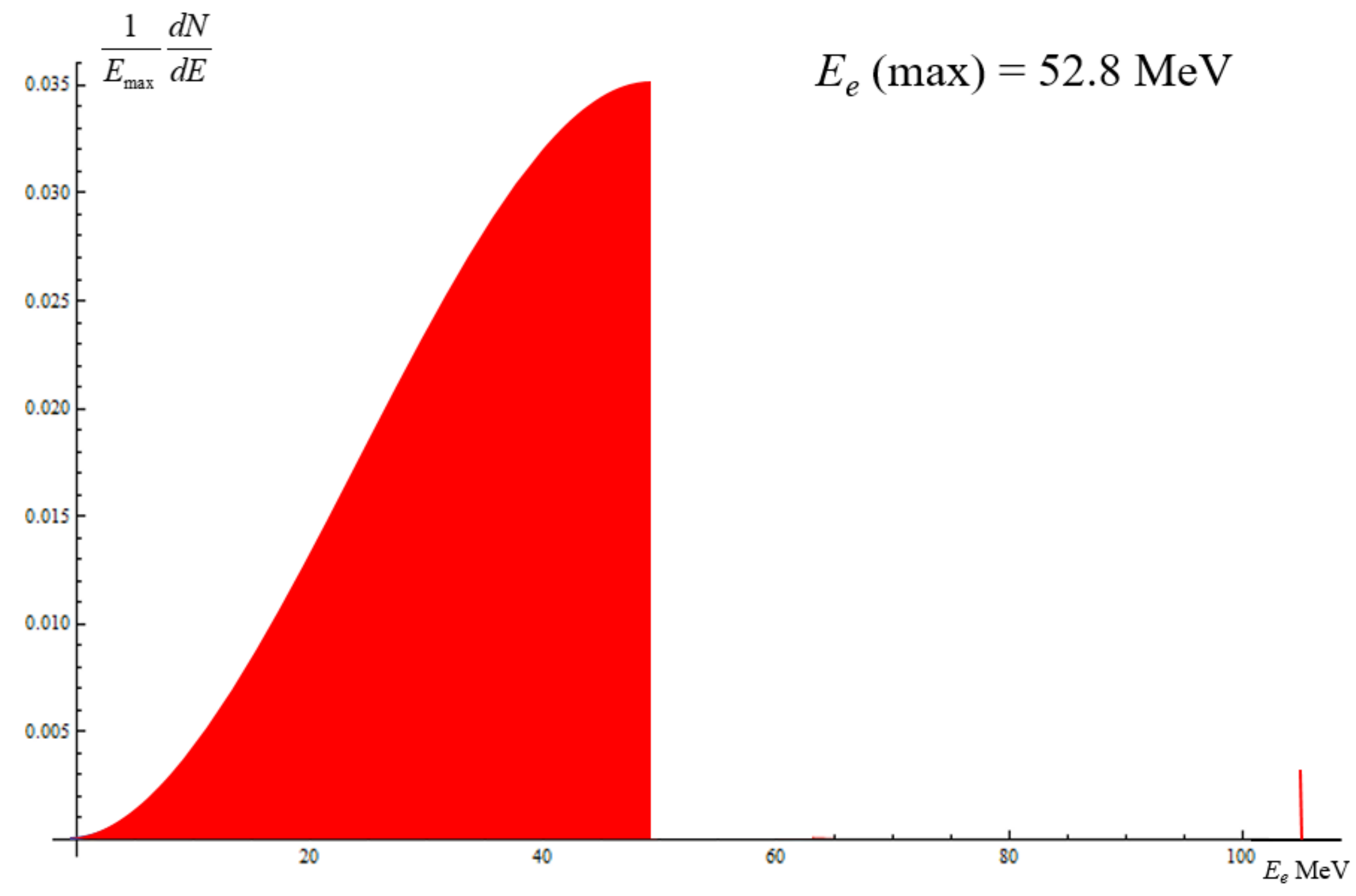




\section{Decay In Orbit Energy Distribution}

- Once you include the effect of the $\mu-\mathrm{Al}^{27}$ binding energy and radiative corrections...

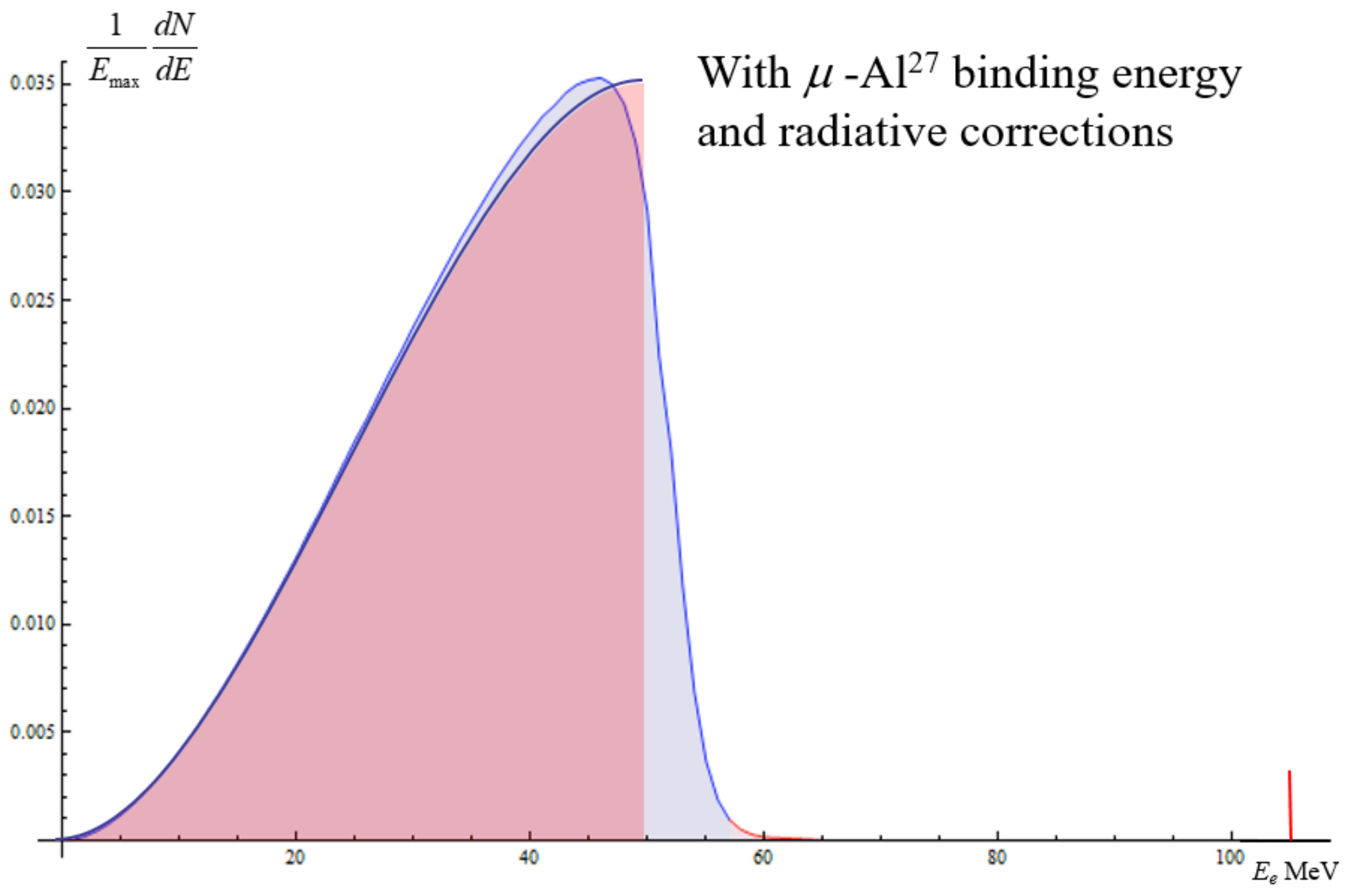




\section{Decay In Orbit Energy Distribution}

- The maximum energy for the DIO electrons comes very close to the signal energy:

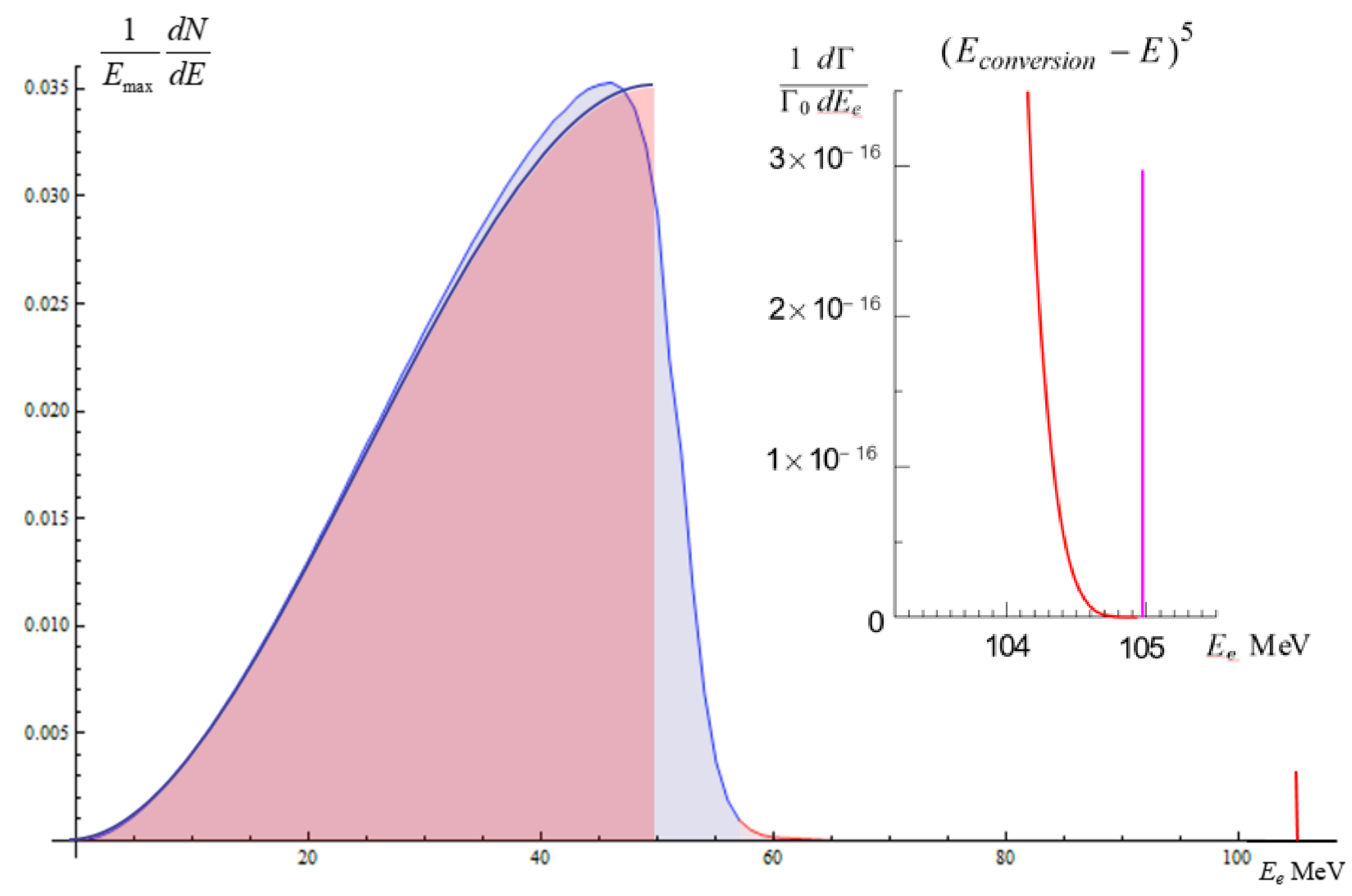

- Therefore, it is important that we have good energy resolution 


\section{Design Overview}

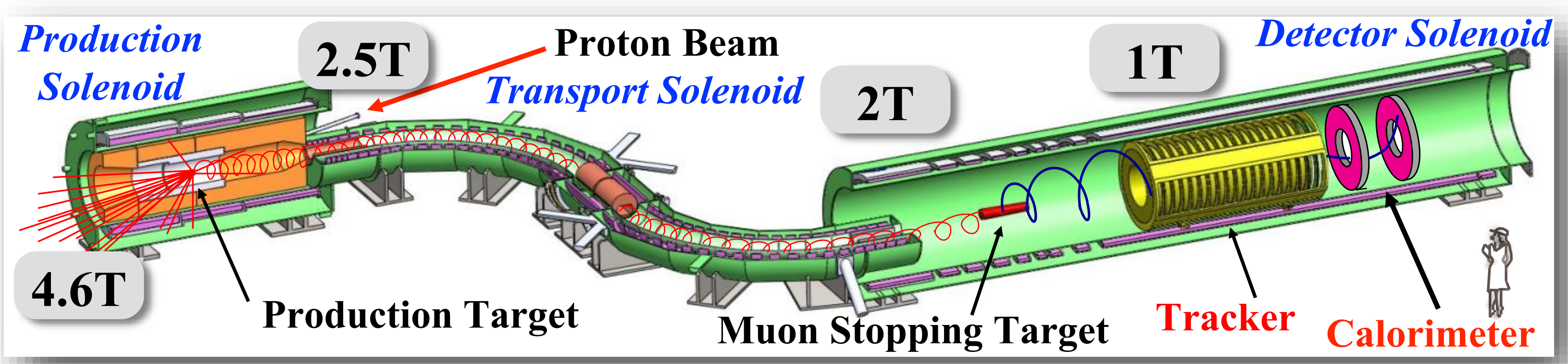

- Production Target + Production Solenoid

- High intensity, pulsed, $8 \mathrm{GeV}$ proton beam strikes tungsten production target producing pions

- Pions are captured by the graded magnetic field and decay to muons

- Transport Solenoid

- Selects low momentum, negative muons

- Absorbers and Collimators eliminate high energy negative particles, positive particles, and line-of-sight neutrals

- Stopping Target, Detector, and Detector Solenoid

- Muons are stopped on an aluminum target

- Tracker measures momentum and trajectories of

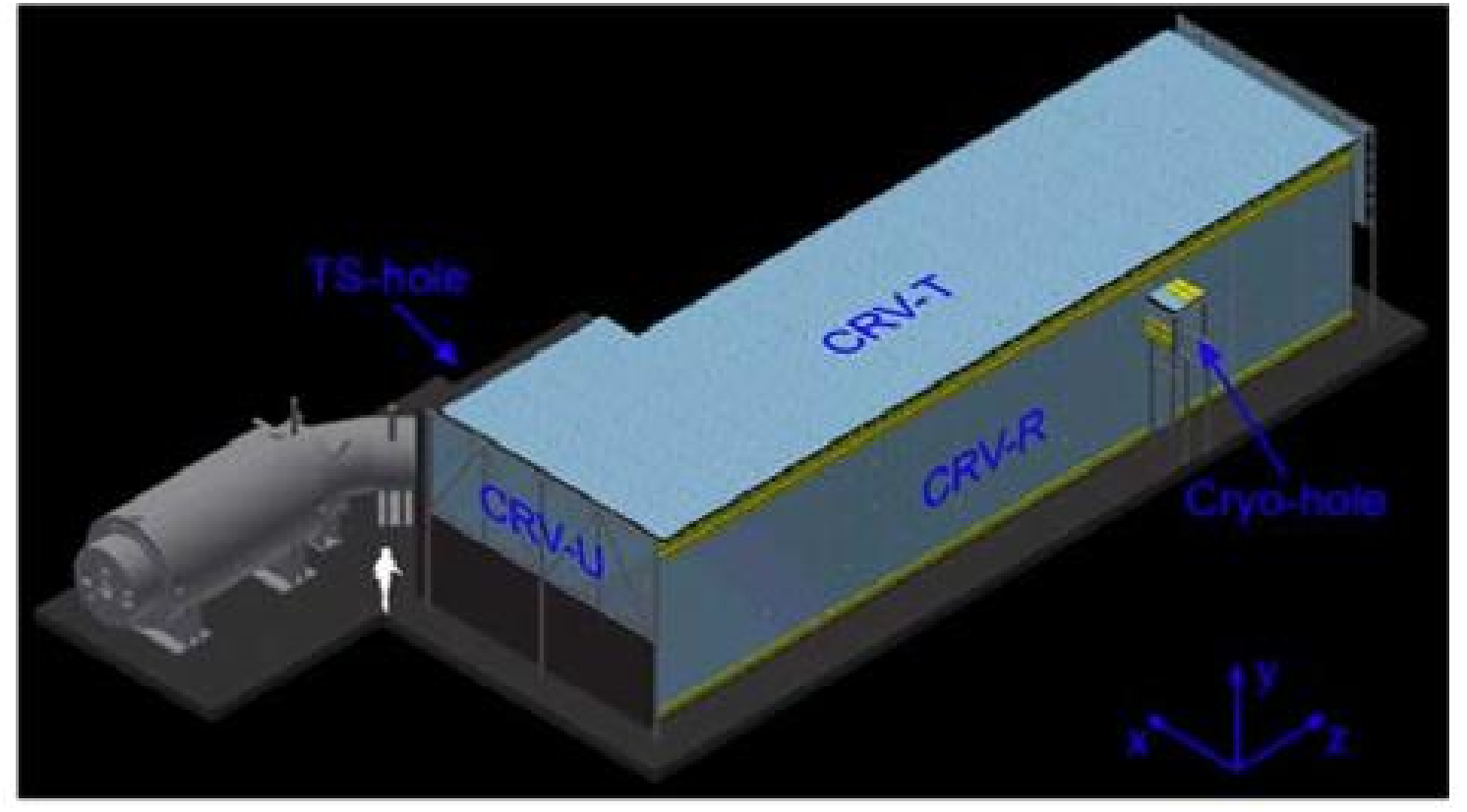
from muonic atoms

- Calorimeter measures energy/time

- Cosmic Ray Veto detector surrounds detector solenoid 


\section{The Mu2e Proton Beam}

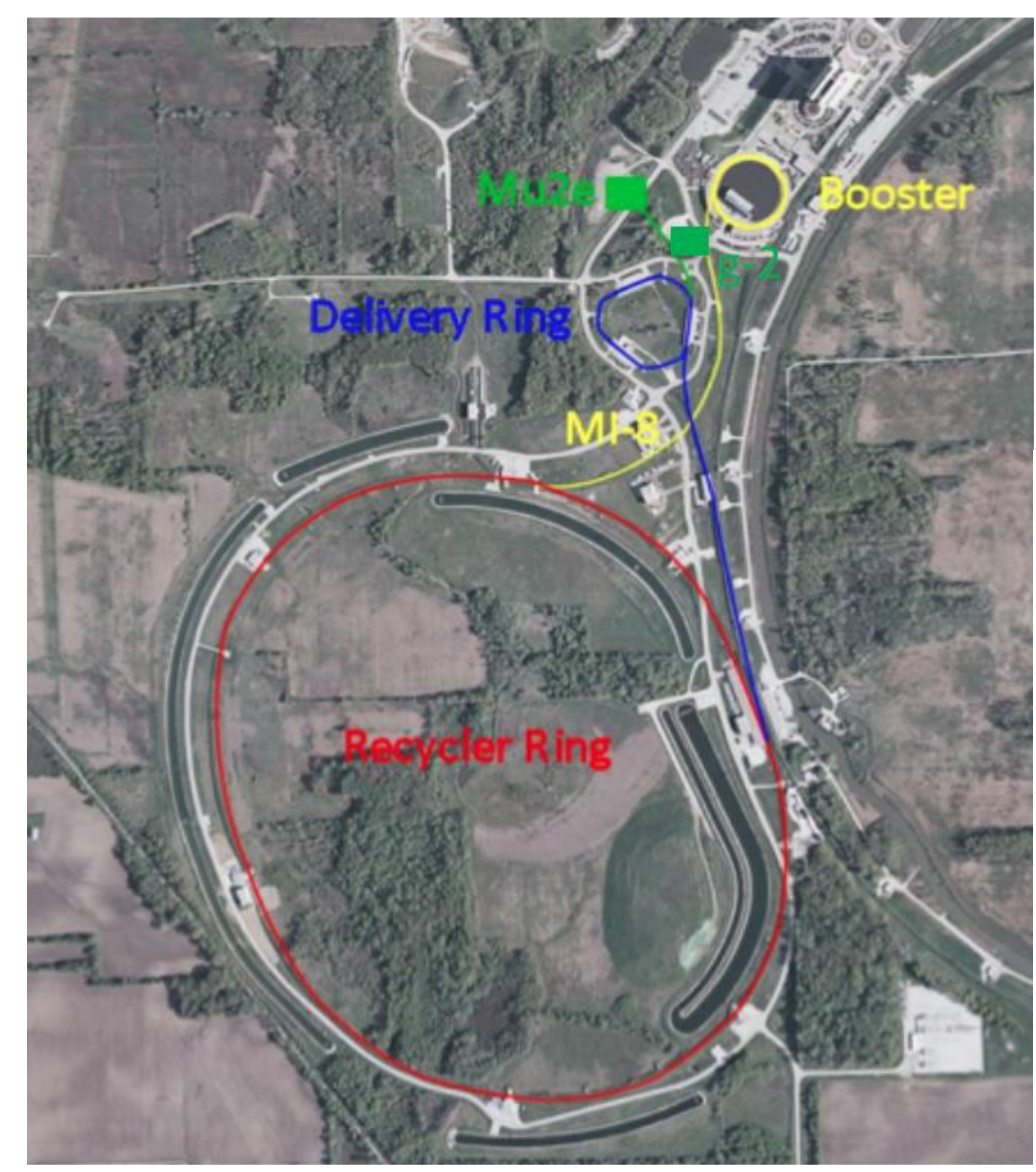

- Mu2e will take advantage of the existing Booster, Recycler, Accumulator, and Antiproton Source Debuncher rings at Fermilab

- Mu2e will run in parallel with NOvA

FERMILAB'S ACCELERATOR CHAIN

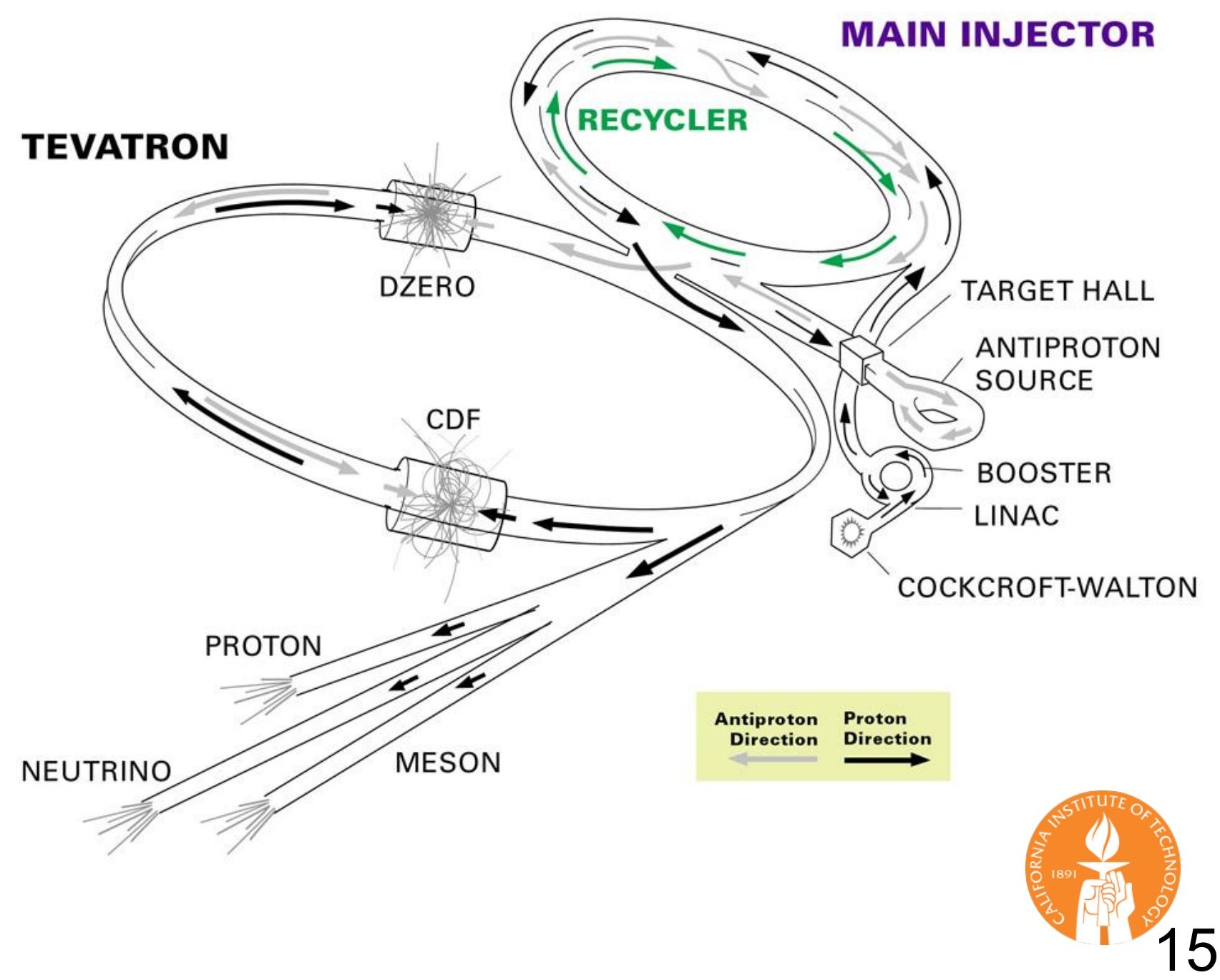




\section{Proton Pulse Structure}

- Proton Pulse Structure:

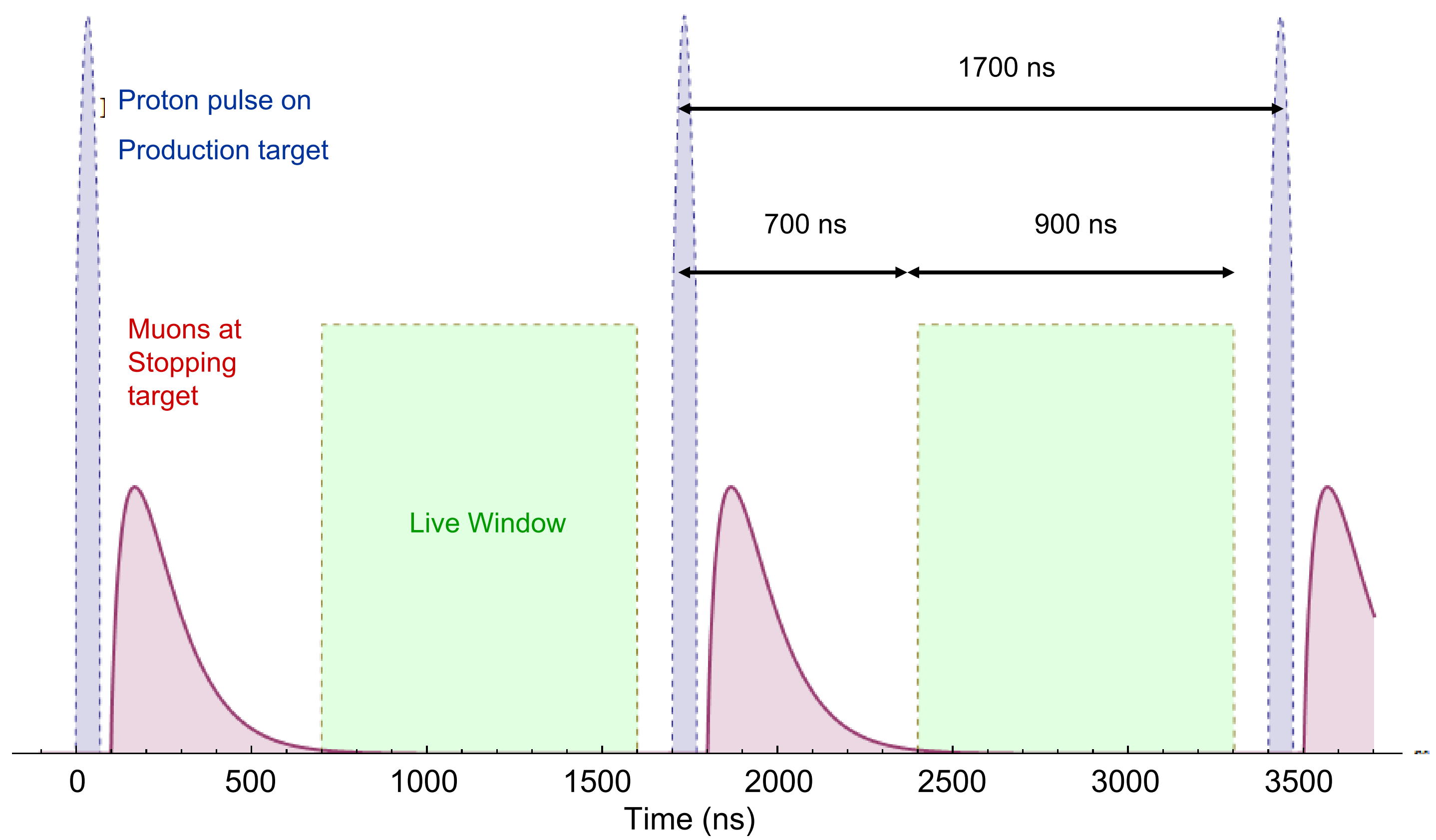

- We use a pulsed proton beam with a delayed data-taking window to suppress short-lived (prompt) backgrounds

- A 700 ns delay reduces pion background by $>10^{-9}$ 


\section{Production Target}

- Production Target

- Radiatively cooled tungsten target suspended by wires

- Produces pions when struck by the proton beam

- Pions are guided to the stopping target by the production and transport solenoids

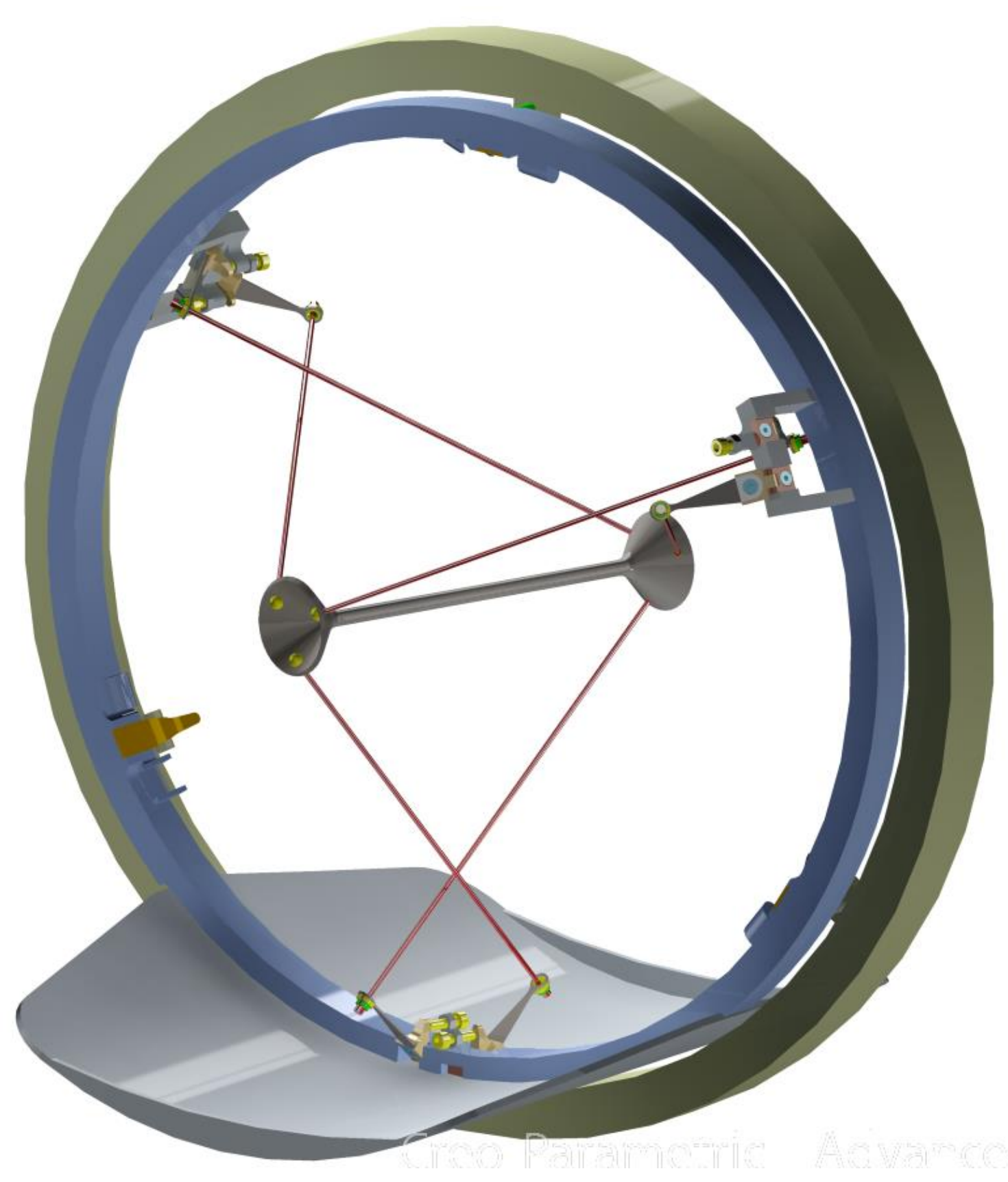




\section{Stopping Target}

- Stopping Target

- Aluminum stopping target composed of foils suspended by wires

- If a signal is seen, other stopping target materials may be used to narrow down what kind of physics is responsible

- Design is still being optimized, but it will probably consist of something like aluminum foil annuli suspended at intervals in a cylindrical volume

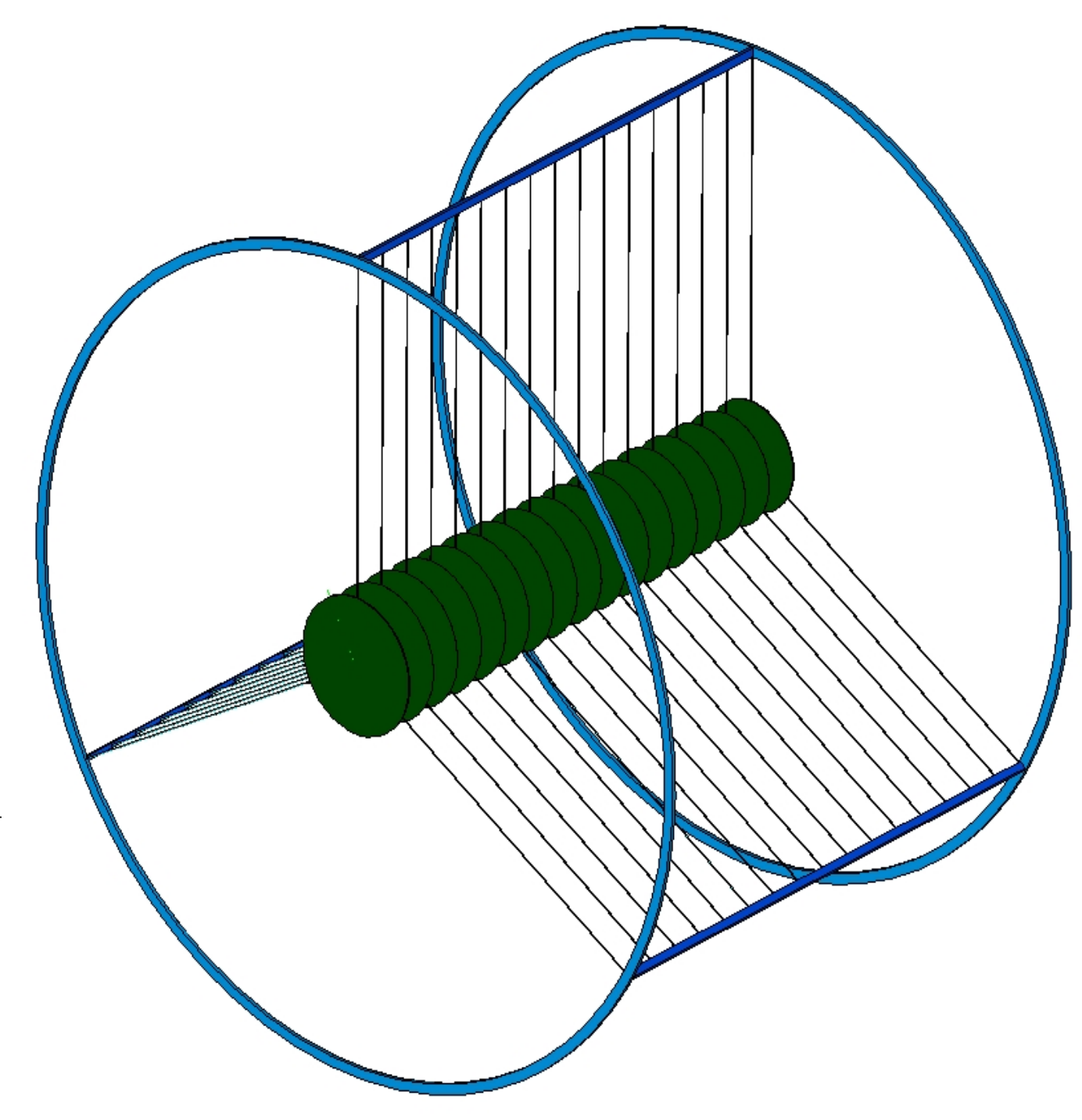




\section{Tracker I}

- A low-mass annular tracker provides us with high-precision measurements of charged particle momenta

- Designed to function in a high background environment

- Within the detector solenoid, track radius is proportional to energy so we use an annular design that only detects particles with large enough radii
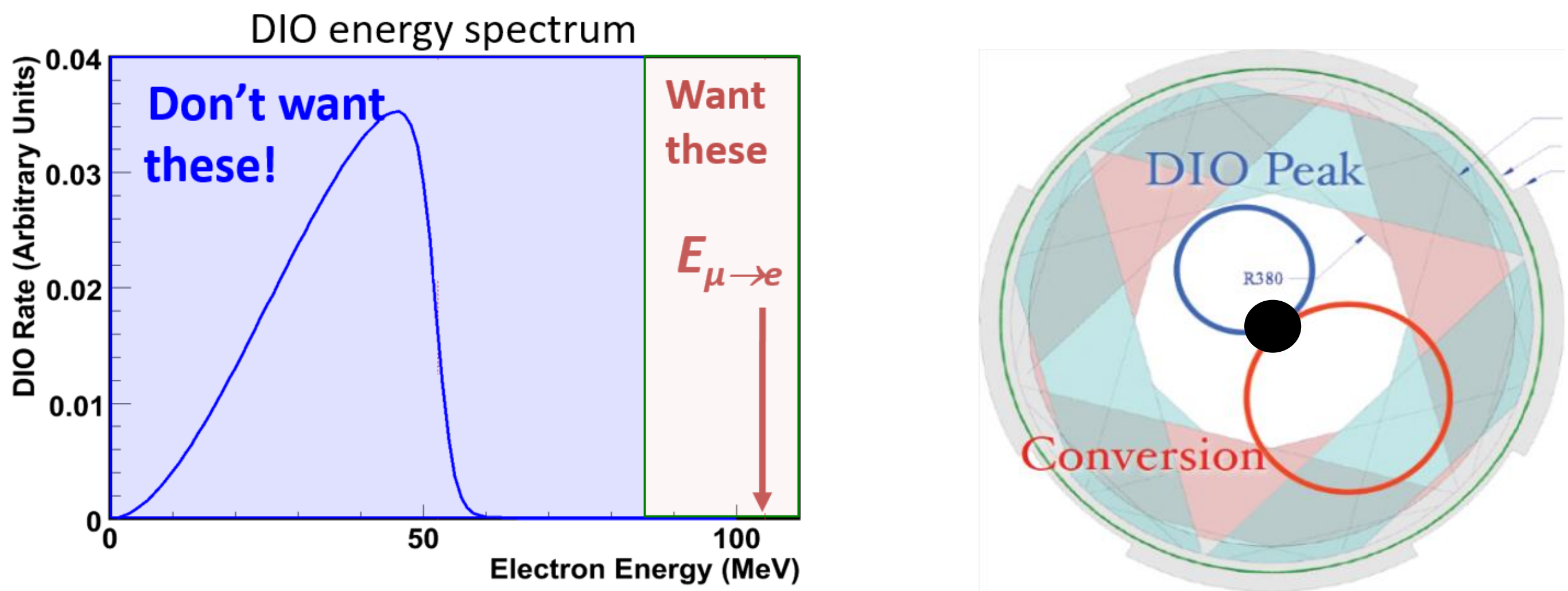

- Expect $<180 \mathrm{keV} / \mathrm{c} p_{T}$ resolution at $105 \mathrm{MeV} / \mathrm{c}(<0.18 \%)$ 


\section{Tracker II}

- Tracker Construction:

- Tracker is constructed from self-supporting panels of low mass straws tubes detectors:

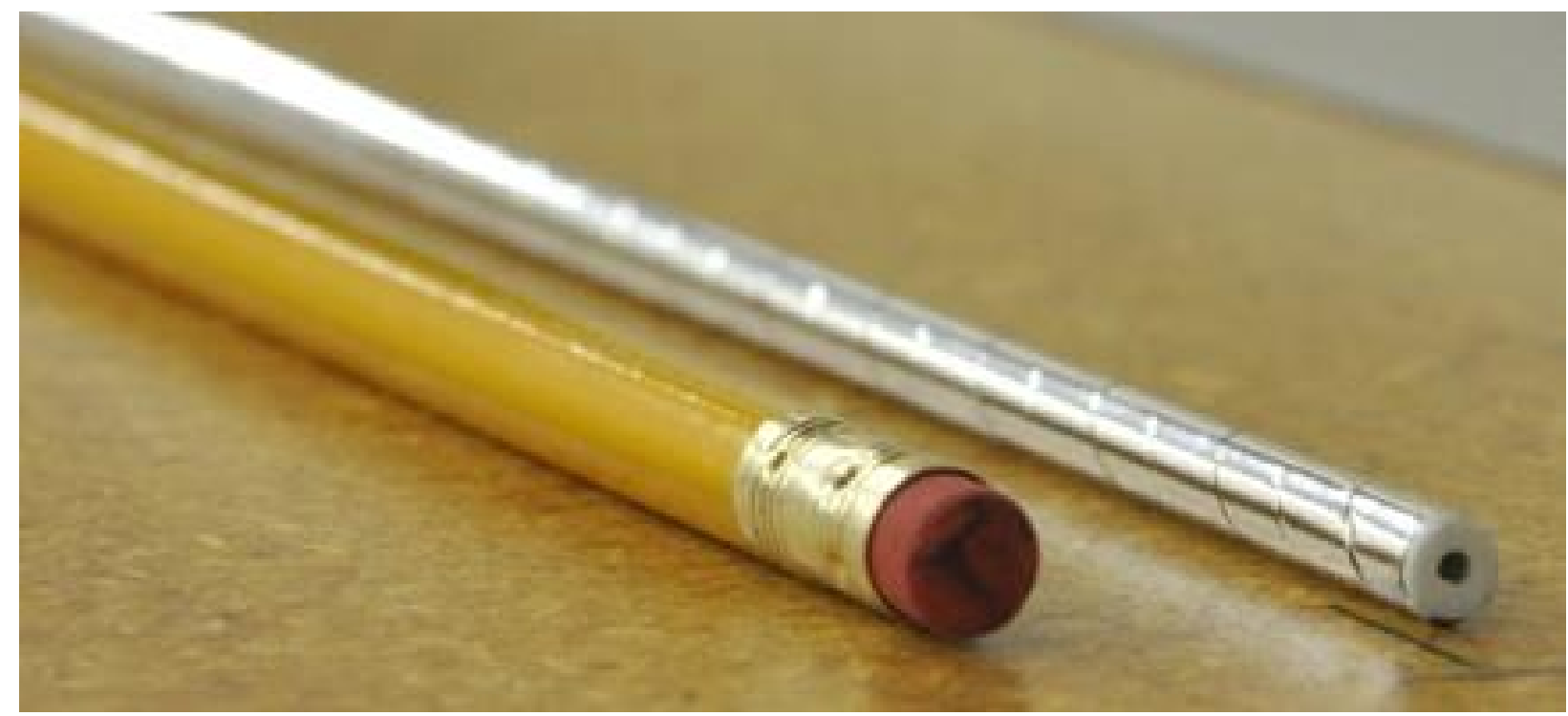

- 5 mm diameter straw

- Spiral wound

- Walls: $12 \mathrm{~mm}$ Mylar $+3 \mathrm{~mm}$ epoxy

$+200 \AA \mathrm{Au}+500 \AA \mathrm{Al}$

- $25 \mathrm{~mm}$ Au-plated W sense wire

- $33-117 \mathrm{~cm}$ in length

- 80/20 Ar/CO2 with HV < $1500 \mathrm{~V}$

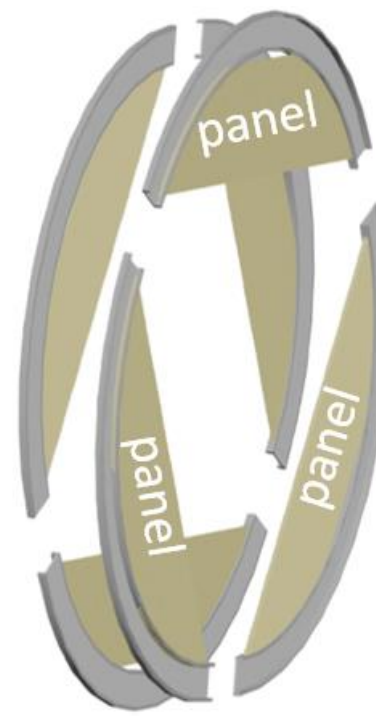

96 straws/panel

- Sets of 6 panels are attached to form a plane, 2 planes are combined to form a station, and 18 stations are arranged in a cylindrical volume to form the tracker:

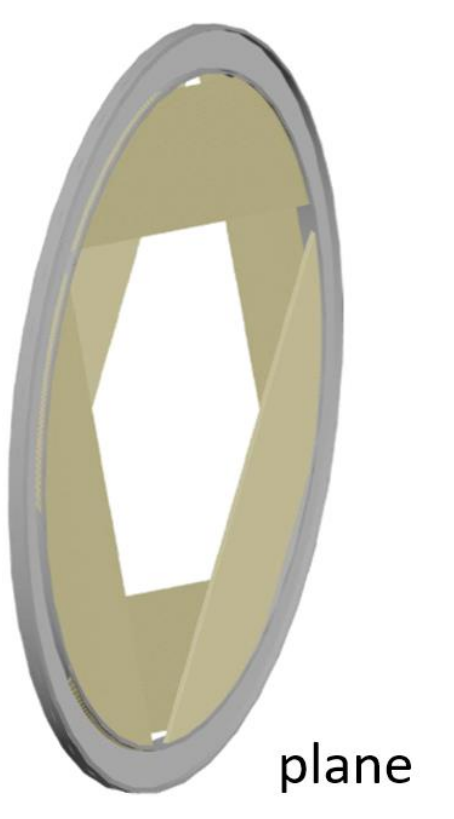

6 panels/plane

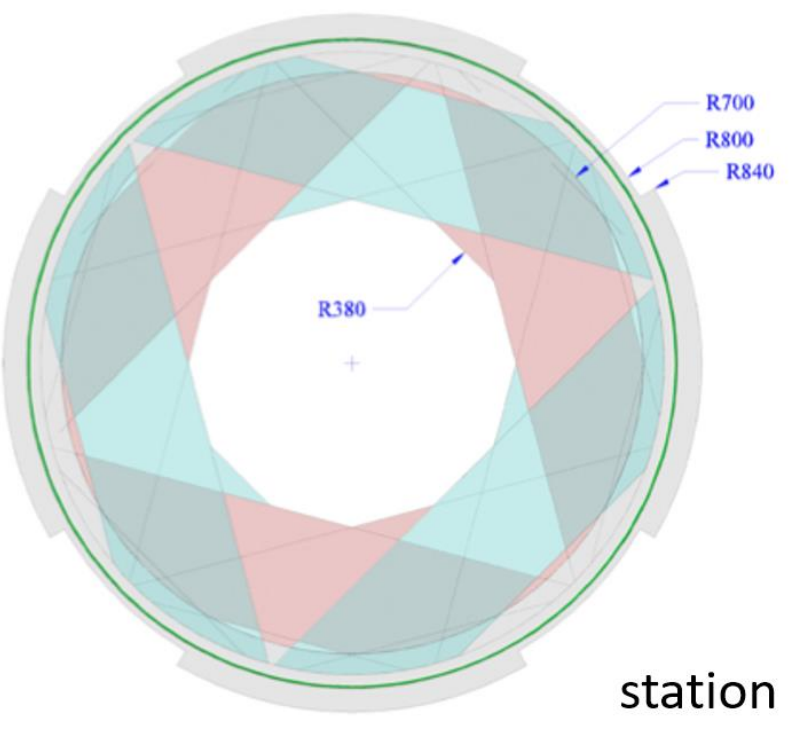

2 panels/station

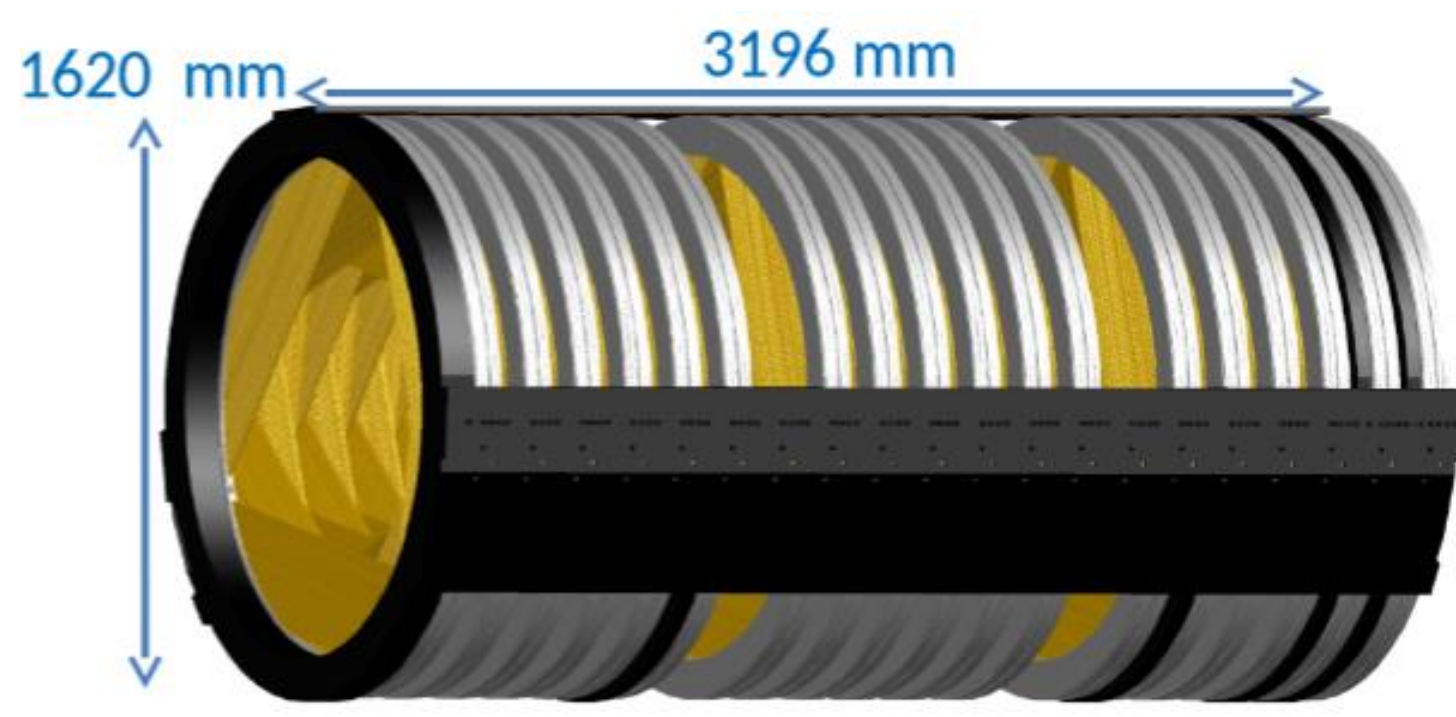

18 station tracker 


\section{Tracker III}

- Tracker Construction:
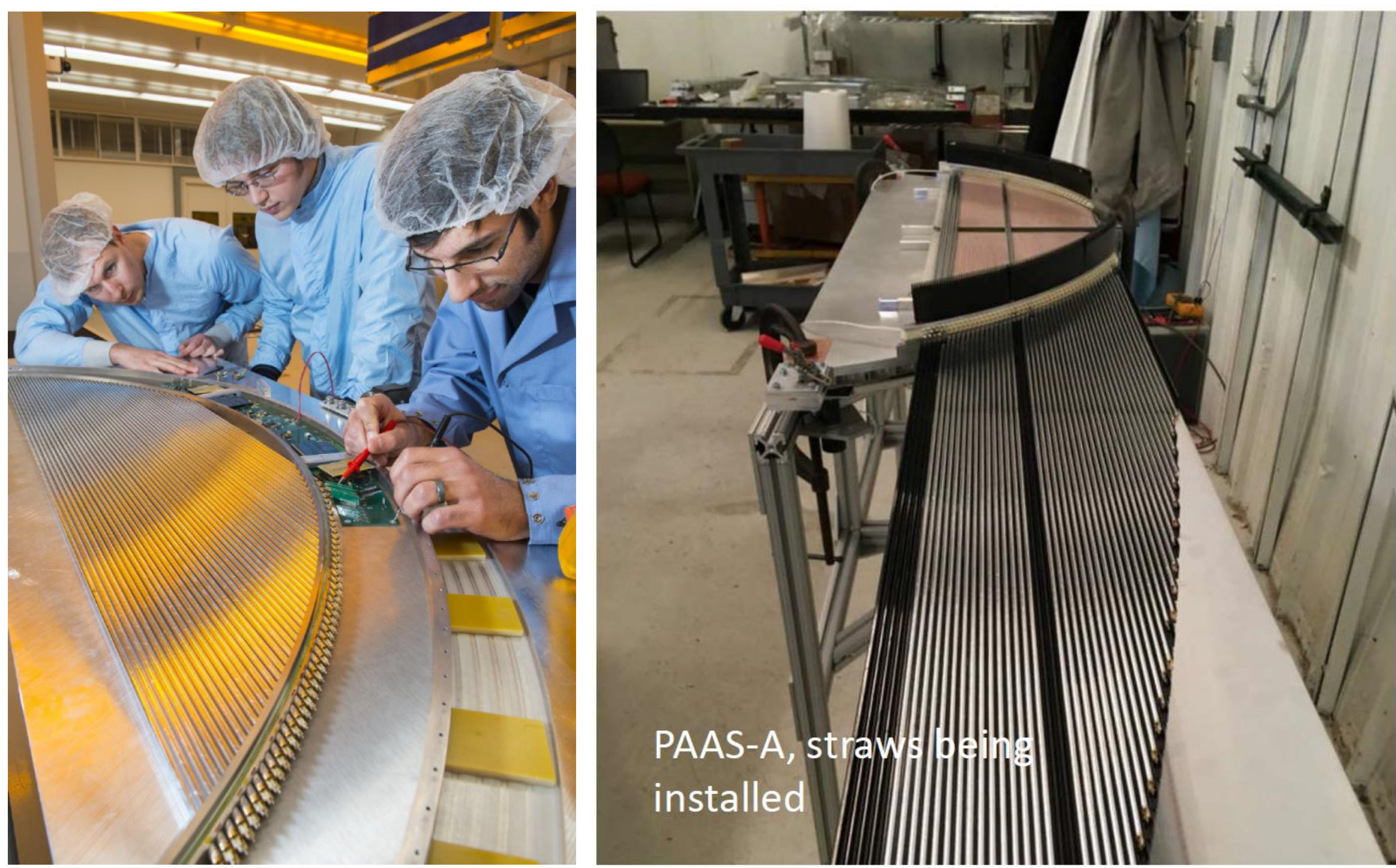


\section{Calorimeter I}

- Calorimeter Serves to

- Distinguish muons from electrons

- Aid in track pattern recognition

- Provide tracker-independent trigger

- Provide accurate timing information for bkg rejection

- Calorimeter Design:

- Two annuli with radius $37-66 \mathrm{~cm}$

- Disks separated by $70 \mathrm{~cm}(1 / 2 \lambda)$

- $\quad$ 674 CsI crystals per disk

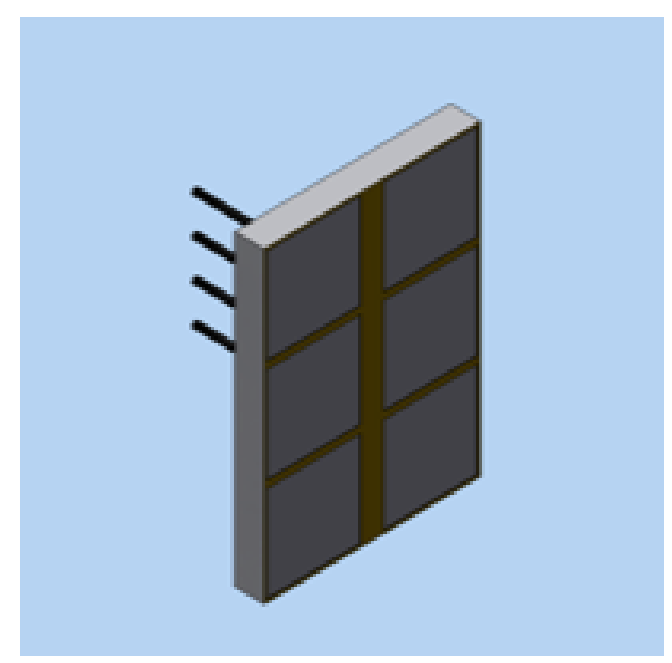

- Two $14 \times 20 \mathrm{~mm}^{2}$ six-element SiPMs / crystal

- Square crystals $\left(34 \times 34 \times 200 \mathrm{~mm}^{3}\right)$
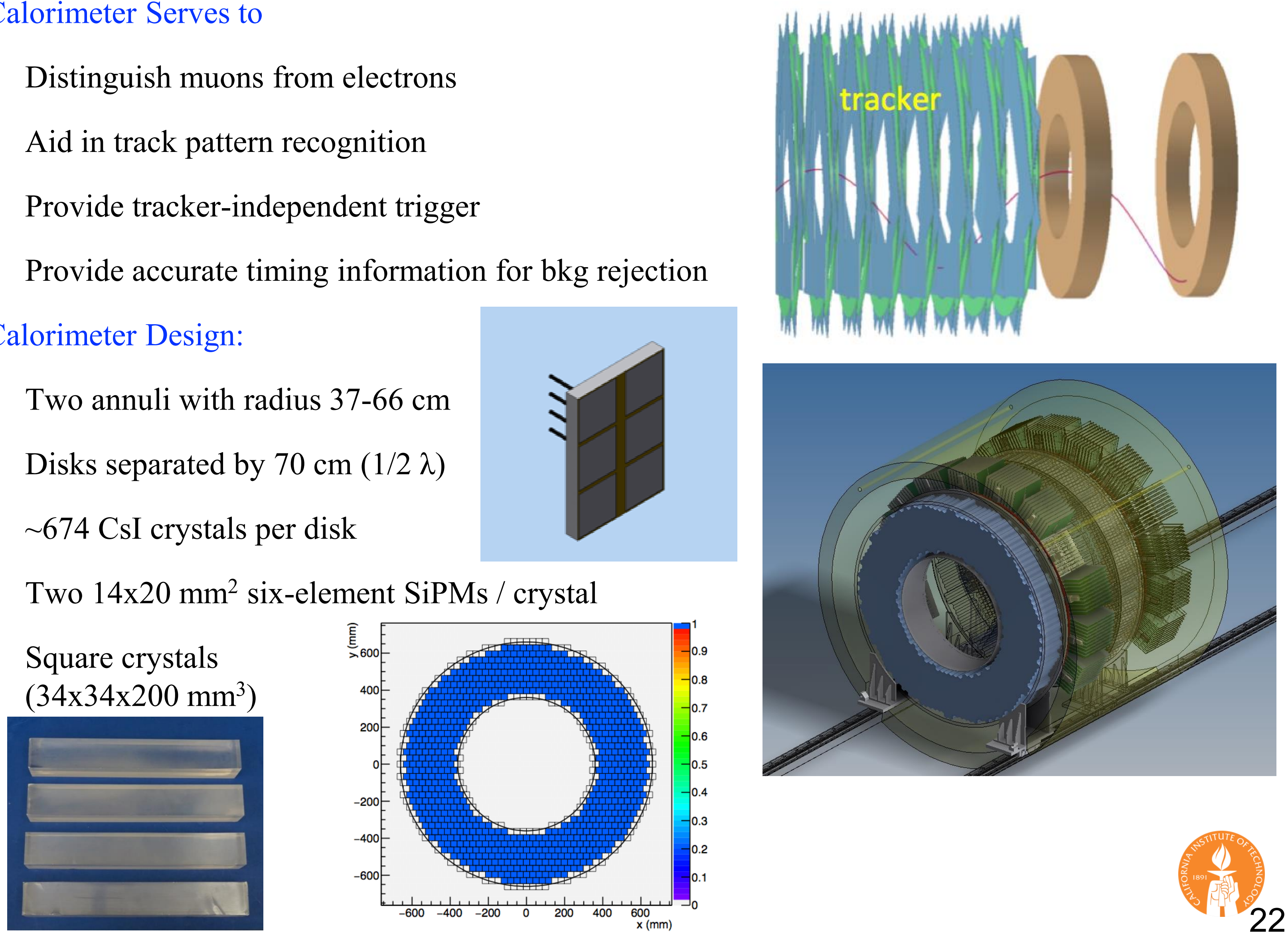


\section{Calorimeter II}

- Wrap crystals in Tyvek and stack in annulus

- A backplane assembly provides cooling and slots mounting crystal readout electronics

- Insert SiPM holders with front end electronics (FEE) into the backplane (air-gap coupling)

- FEE are read out by readout controllers housed in crates
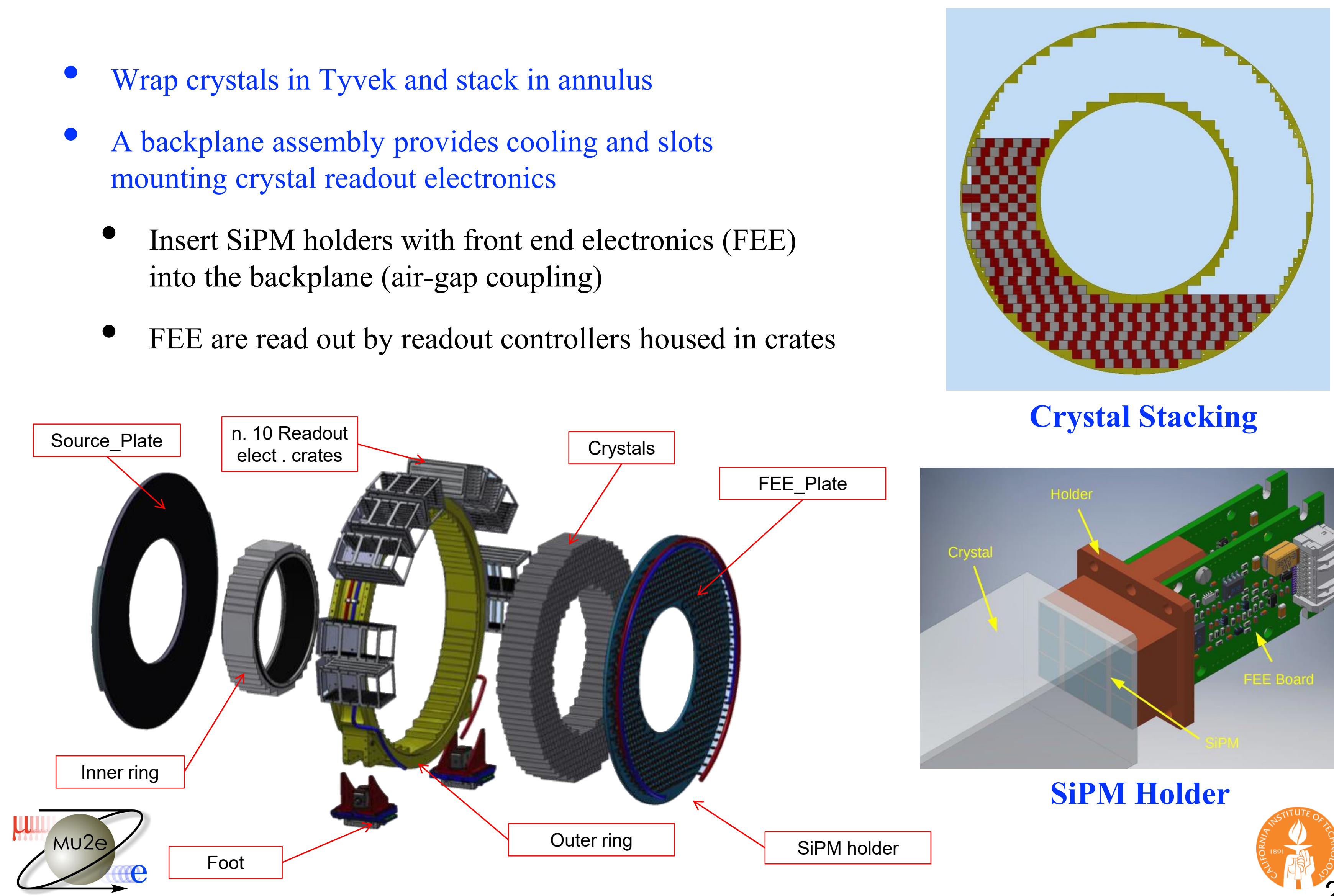

Crystal Stacking

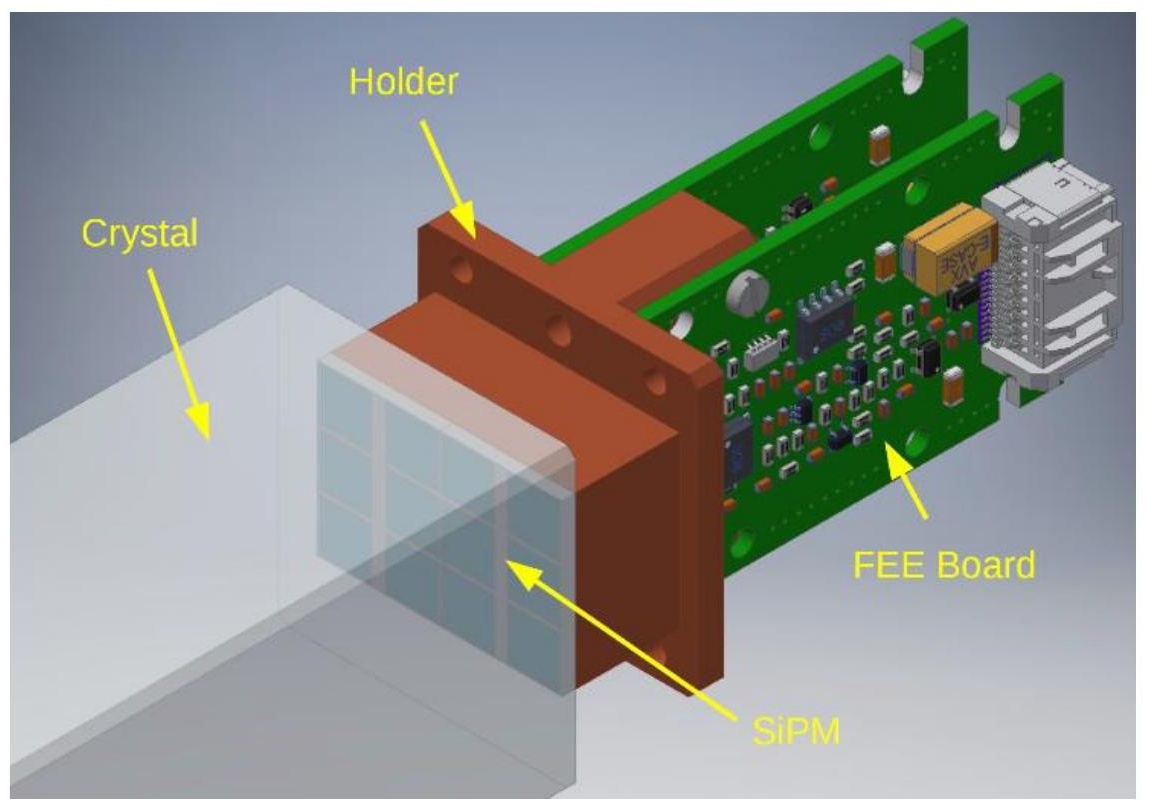

SiPM Holder 


\section{Calorimeter III}

- The calorimeter will be calibrated using activated Fluorine-rich fluid

- Fluorinert is activated using neutrons from a DT generator

- Fluid is pumped through pipes in front of the disks

- Calibrate energy scale to $<0.5 \%$ in a few minutes

- A UV laser system will continuously monitor SiPM gains

- Distribute light using silica optical fibers
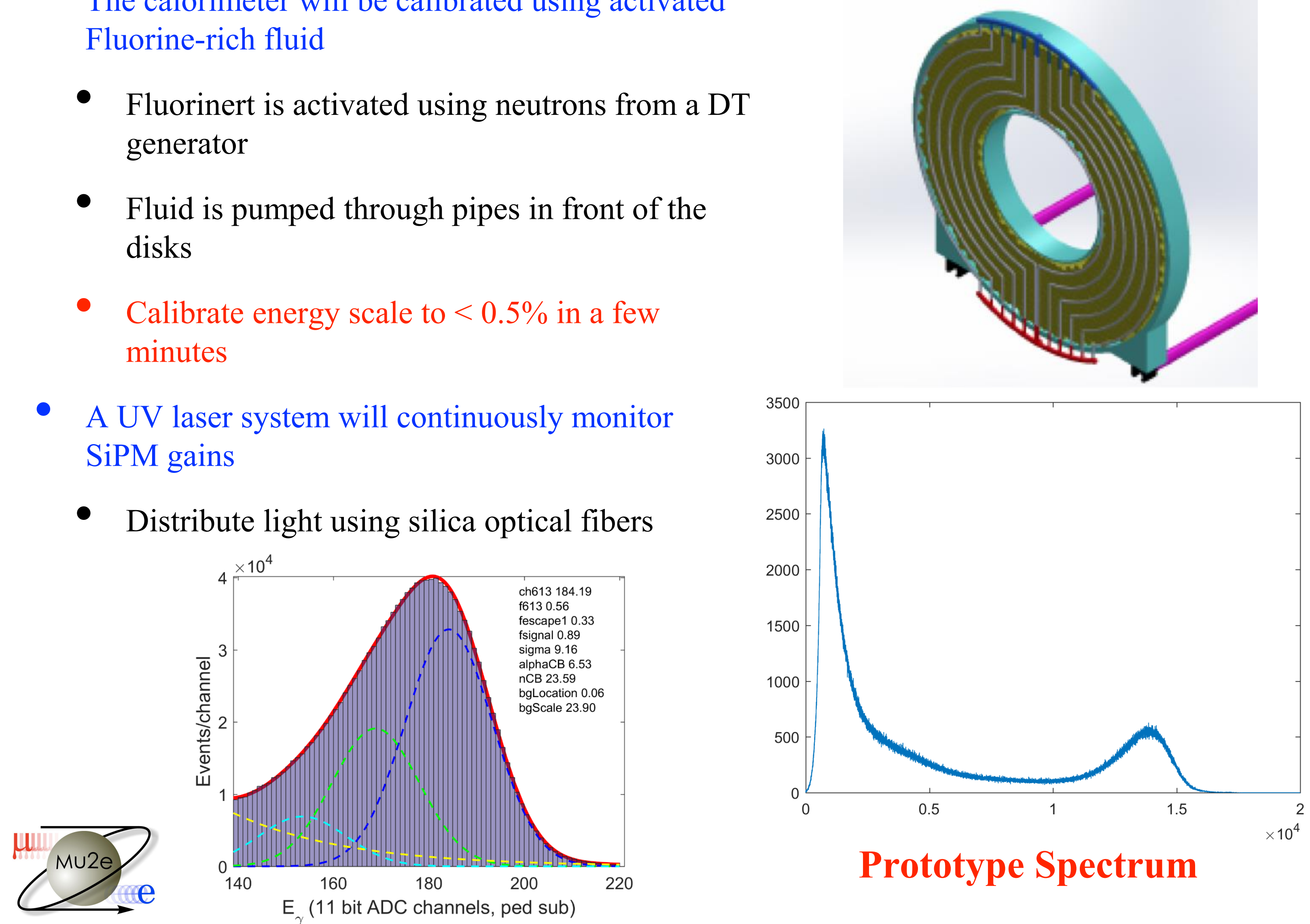

Prototype Spectrum 


\section{Calorimeter Prototype}
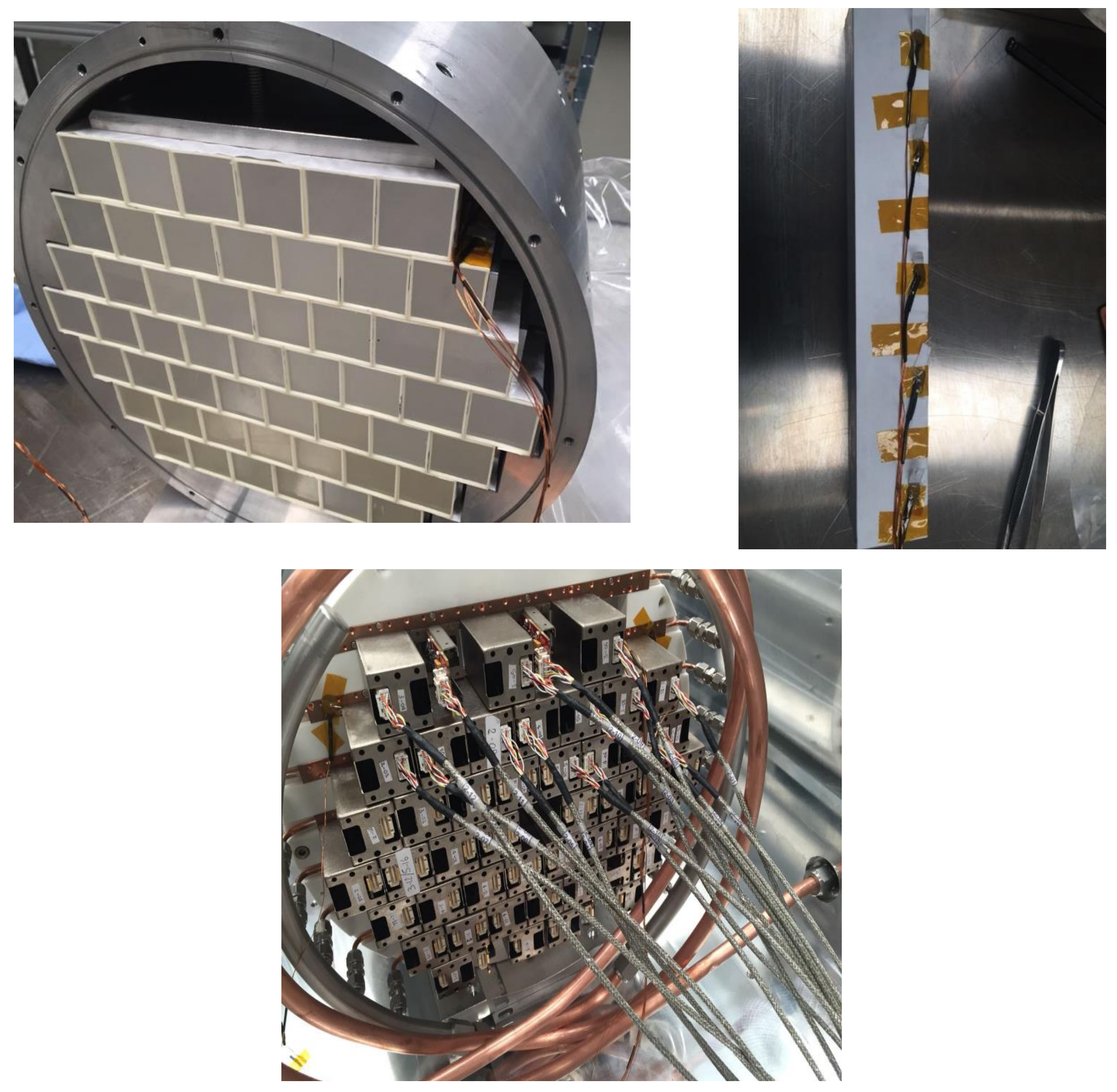


\section{Calorimeter Prototype Test Beam}

- May 2017 test beam with 70-115 MeV electrons at INFN Frascati

- $5130 \times 30 \times 200 \mathrm{~mm}^{3}$ CsI crystals

- Readout: Hamamatsu, SNESL, and Advansid MPPCs

- Results:

\section{ENERGY RESOLUTION}

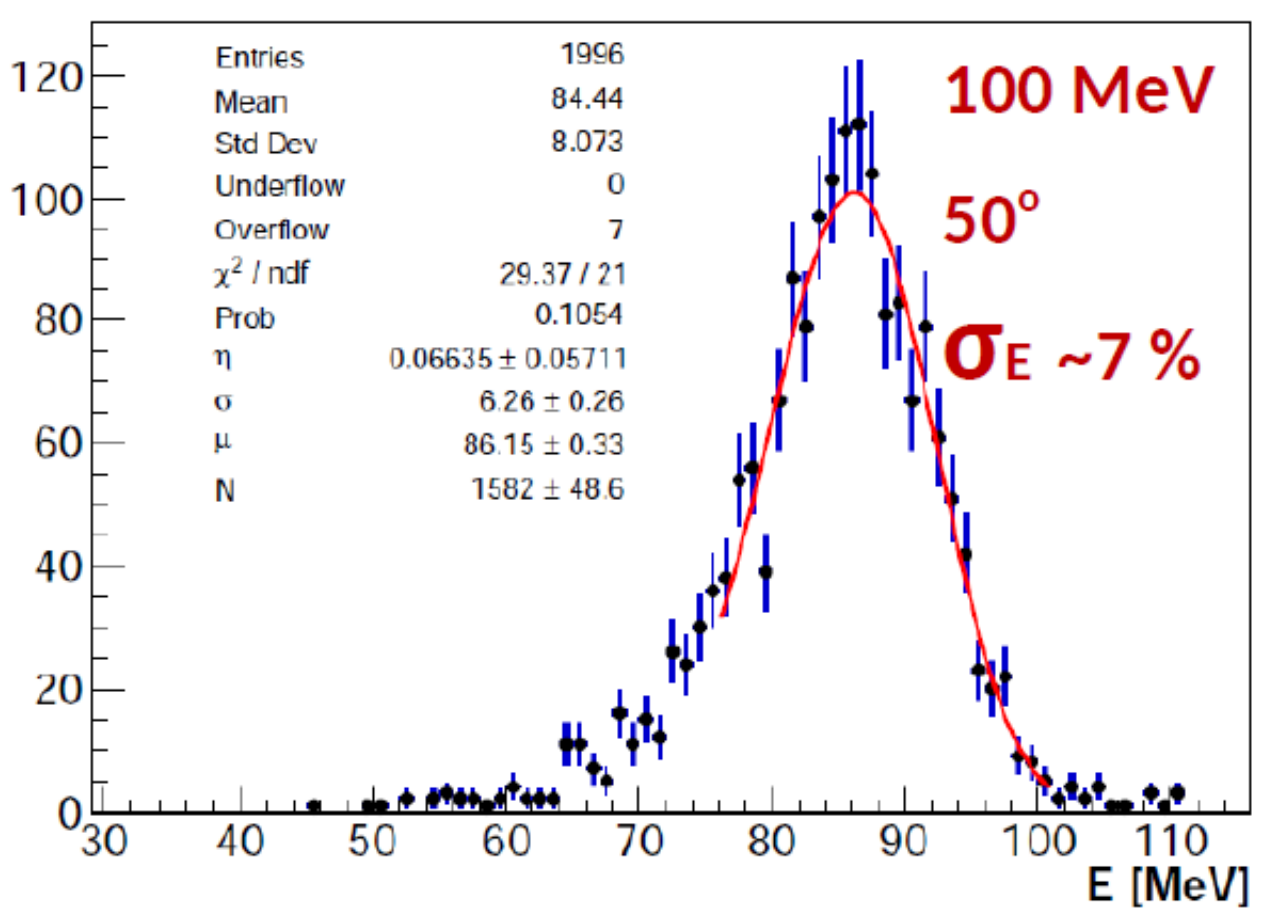

Calibration with $\mathrm{e}^{-}$at $0^{\circ}$

For $100 \mathrm{MeV}^{-}$at $50^{\circ}$ : Energy resolution $\sim 7 \%$

\section{TIME RESOLUTION}

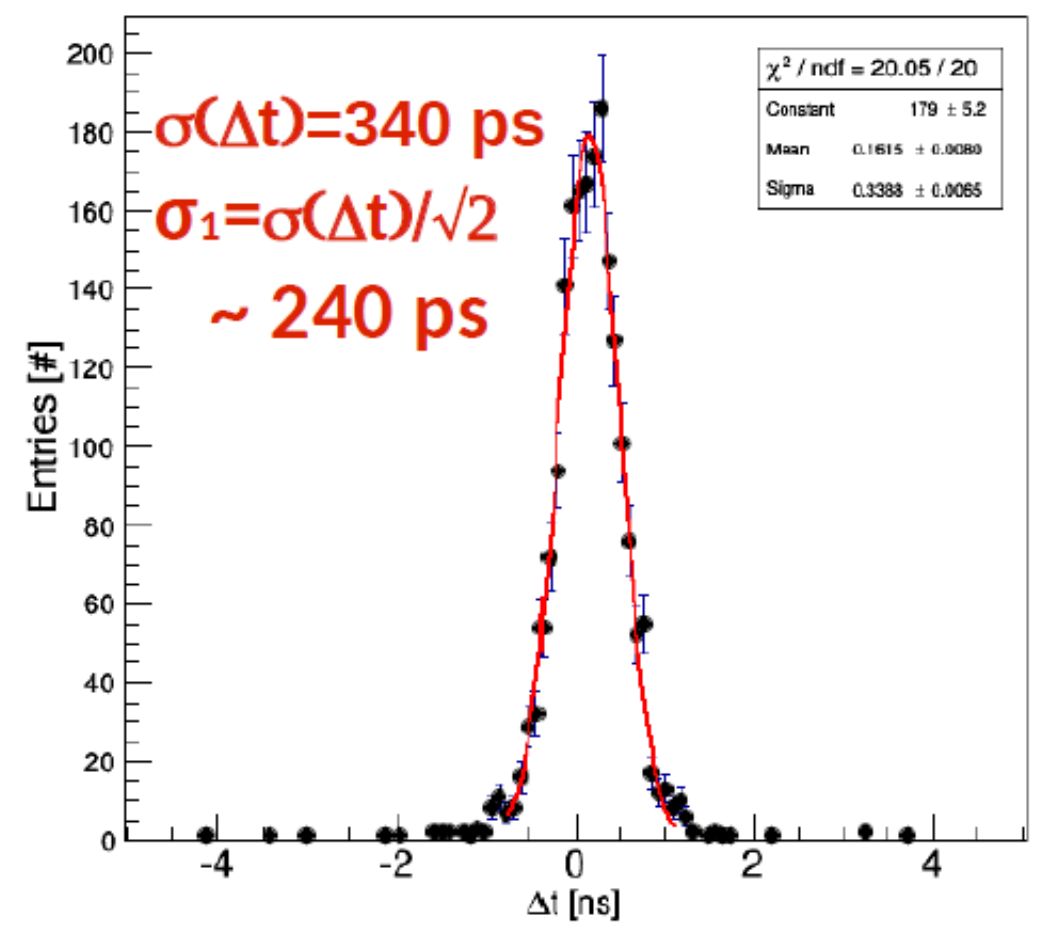

$\Delta \mathrm{t}$ : Time difference between two sensors reading the same crystal.
For $100 \mathrm{MeV} \mathrm{e}^{-}$at $50^{\circ}$ :

$\Delta$ t resolution $\sim 340$ ps

Single sensor resolution $\sim 240$ ps

- Energy and time resolutions are well within the requirements 


\section{Cosmic Ray Veto I}

- The Cosmic Ray Veto (CRV) system surrounds the detector solenoid and half the transport solenoid

- CRV identifies cosmic ray muons

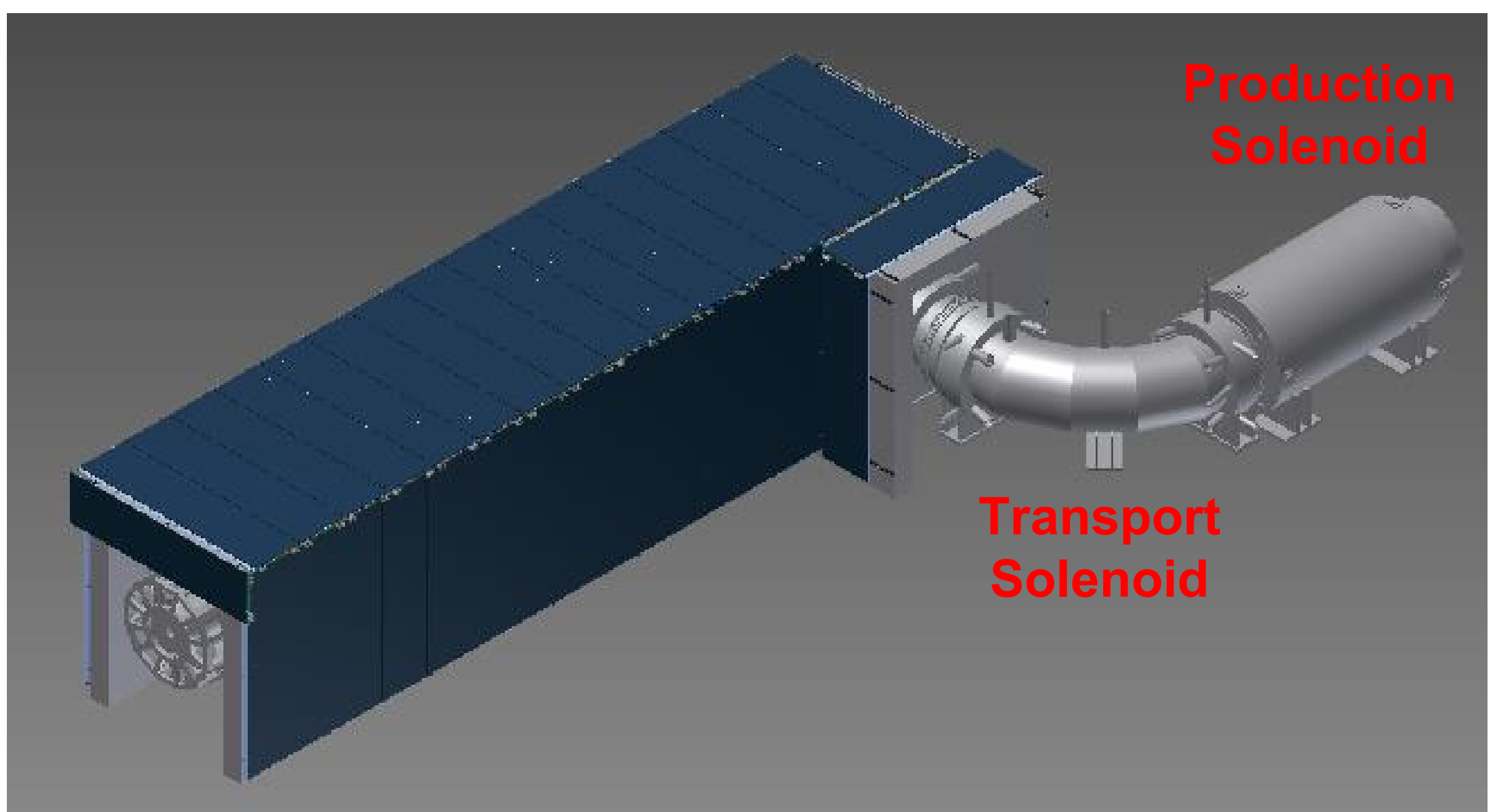

- Each day, $\sim 1$ conversion-like electron is produced by cosmic rays

- Need the CRV to suppress this background 


\section{Cosmic Ray Veto II}

- The CRV is composed of 4 layers of overlapping panels of extruded polystyrene scintillator
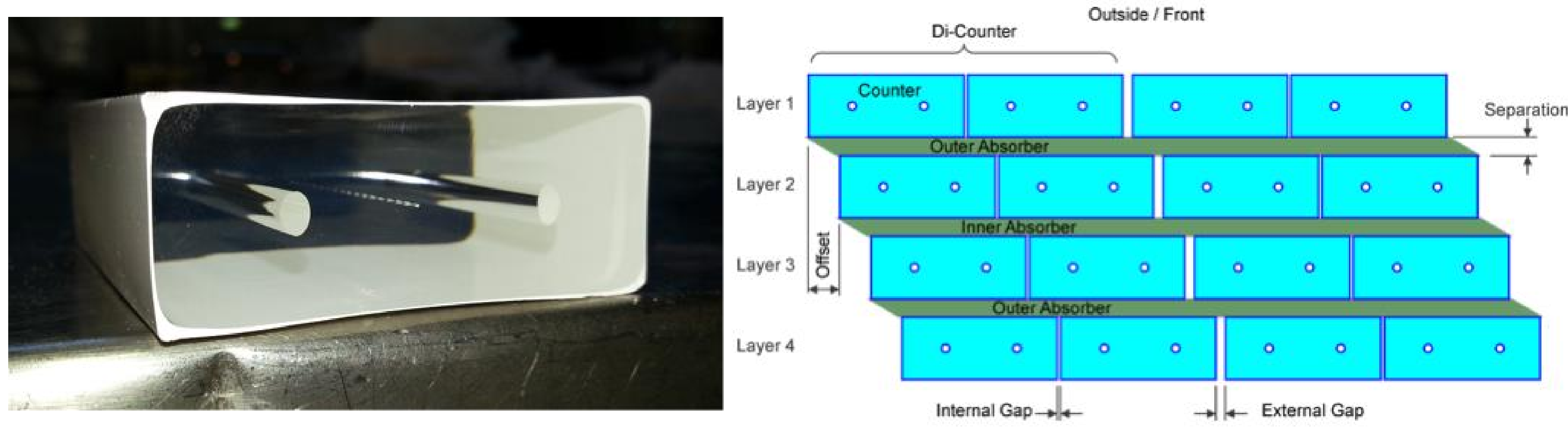

- Each panel is composed of $5 \times 2 \times \sim 450 \mathrm{~cm}^{3}$ scintillator bars

- 2 embedded wavelength-shifting fibers per bar

- Both ends of the bars are readout by SiPMs

- In testing, the veto achieves $\varepsilon>99.4 \%$ per layer 


\section{DAQ/Trigger}

- Data Acquisition (DAQ) system provides readout and control for all the detector subsystems

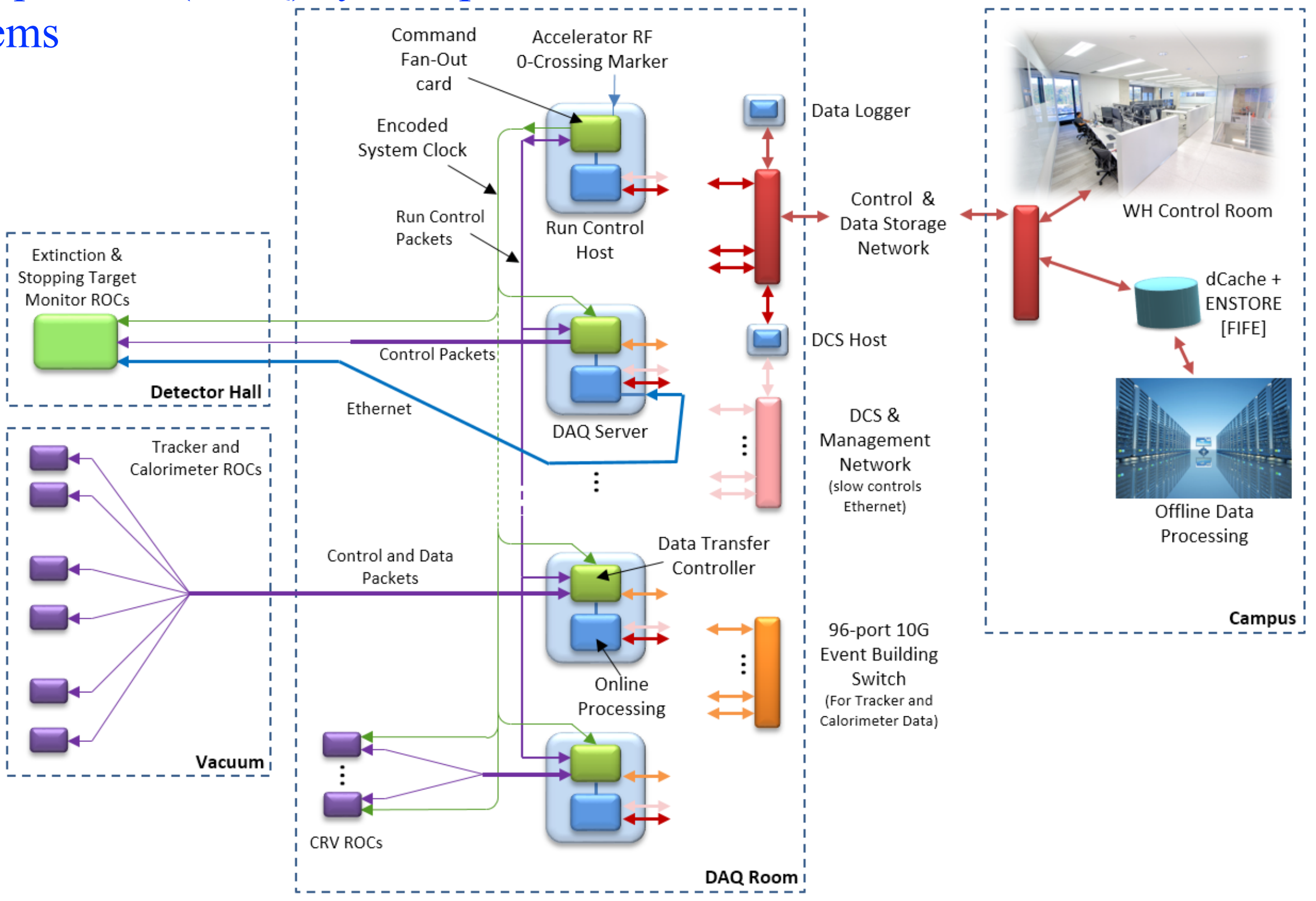

- Trigger processing is handled almost entirely in software (with some FPGA-based pre-processing)

- Allows us to take advantage of commercial computing hardware

- Filters designed and tested in the offline environment can be run in the online trigger environment 


\section{DAQ Server Setup}

- Online processing provided by 40 commercial $3 \mathrm{U}$ rack-mount servers

- Each server houses 2 PCIe cards with onboard FPGA and custom firmware that provide detector readout/control as well as data pre-processing

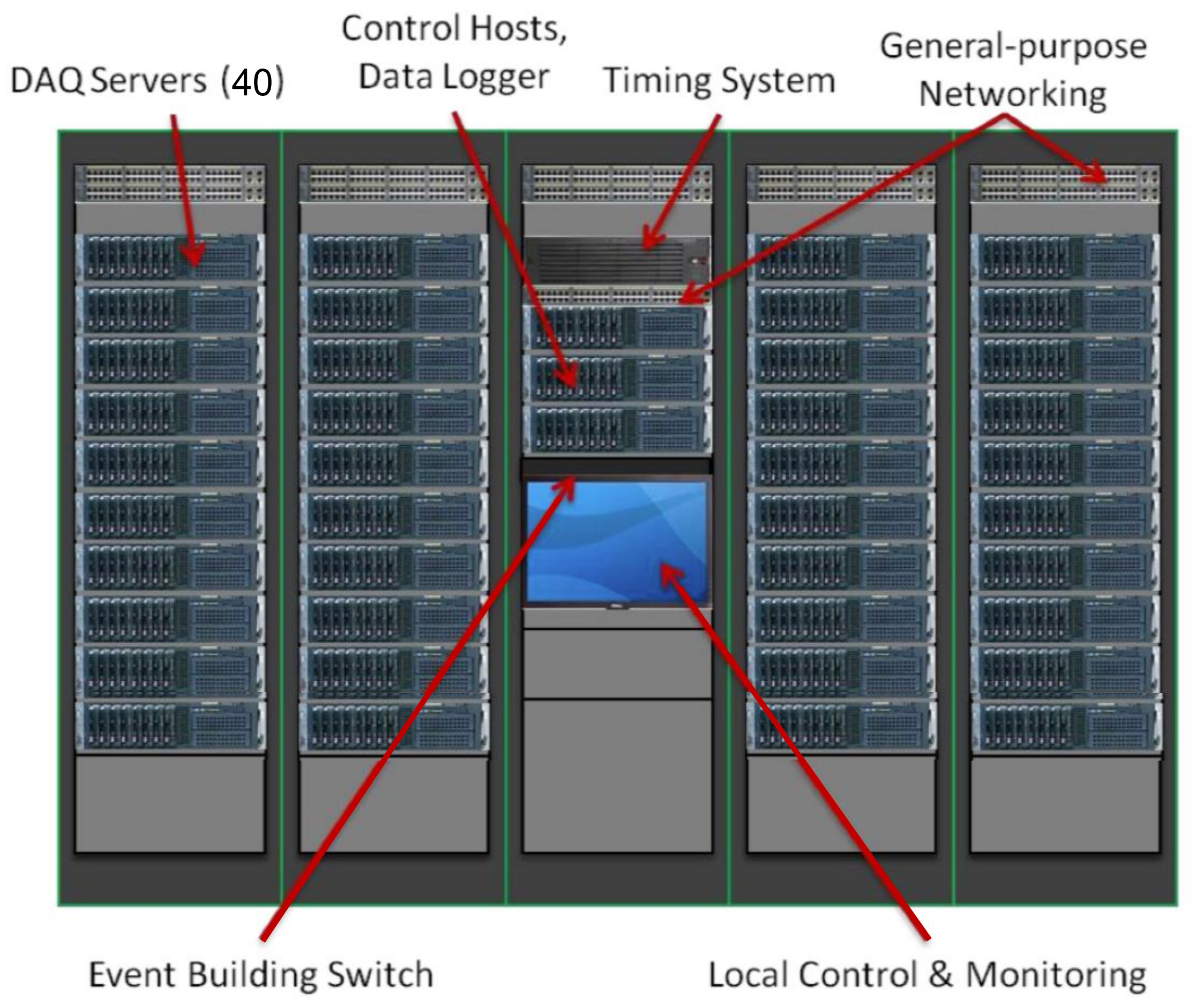




\section{Mu2e Building}

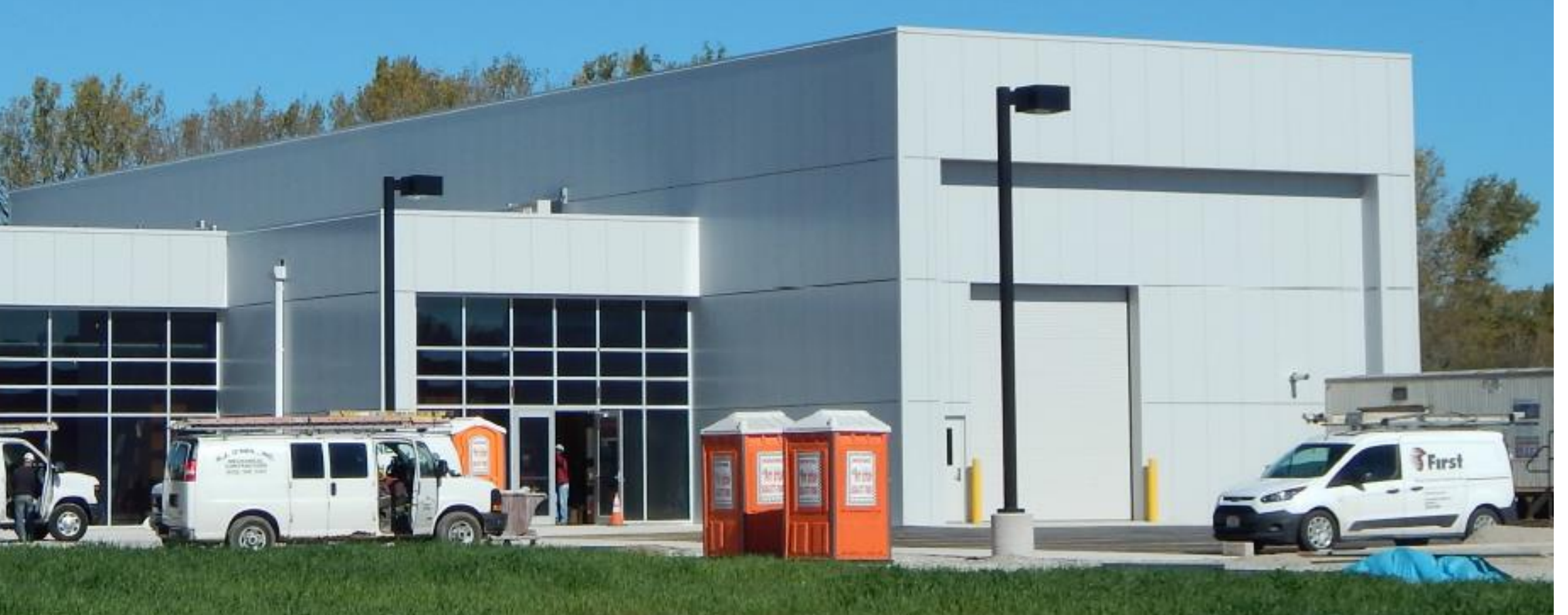

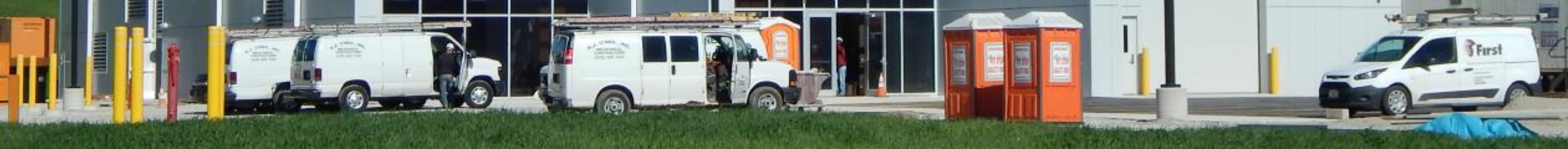

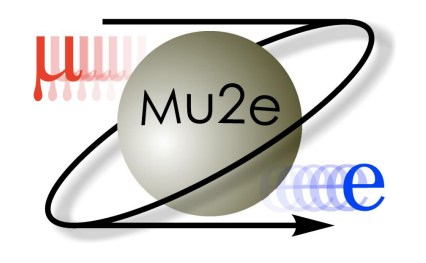




\section{The Mu2e Collaboration}

\section{Over 200 Scientists from 37 Institutions}
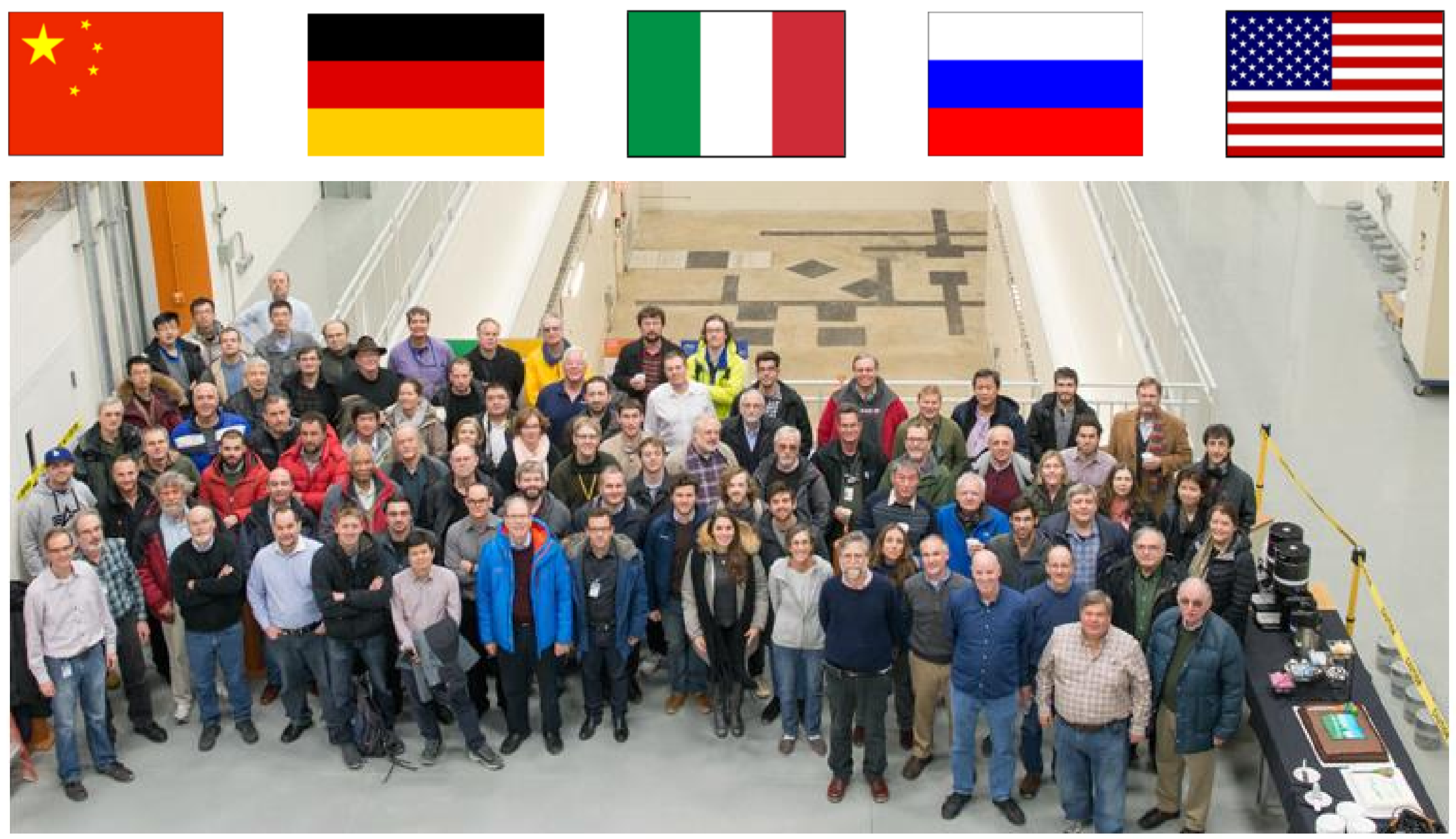

Argonne National Laboratory $\bullet$ Boston University Brookhaven National Laboratory Lawrence Berkeley National Laboratory and University of California, Berkeley - University of California, Davis • University of California, Irvine • California Institute of Technology • City University of New York $\bullet$ Joint Institute for Nuclear Research, Dubna $\bullet$ Duke University $\bullet$ Fermi National Accelerator Laboratory $\bullet$ Laboratori Nazionali di Frascati $\bullet$ INFN Genova $\bullet$ HelmholtzZentrum Dresden-

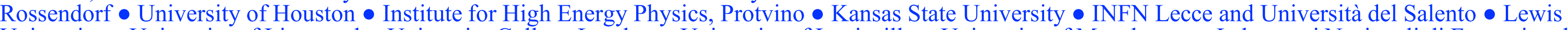
University $\bullet$ University of Liverpool • University College London $\bullet$ University of Louisville $\bullet$ University of Manchester $\bullet$ Laboratori Nazionali di Frascati and Università Marconi Roma $\bullet$ University of Minnesota $\bullet$ Institute for Nuclear Research, Moscow $\bullet$ Muons Inc. $\bullet$ Northern Illinois University $\bullet$ Northwestern University • Novosibirsk State University/Budker Institute of Nuclear Physics • INFN Pisa • Purdue University • University of South Alabama • Sun Yat Sen University • University of Virginia $\bullet$ University of Washington $\bullet$ Yale University 


\section{Detector Hall (Lower Level)}

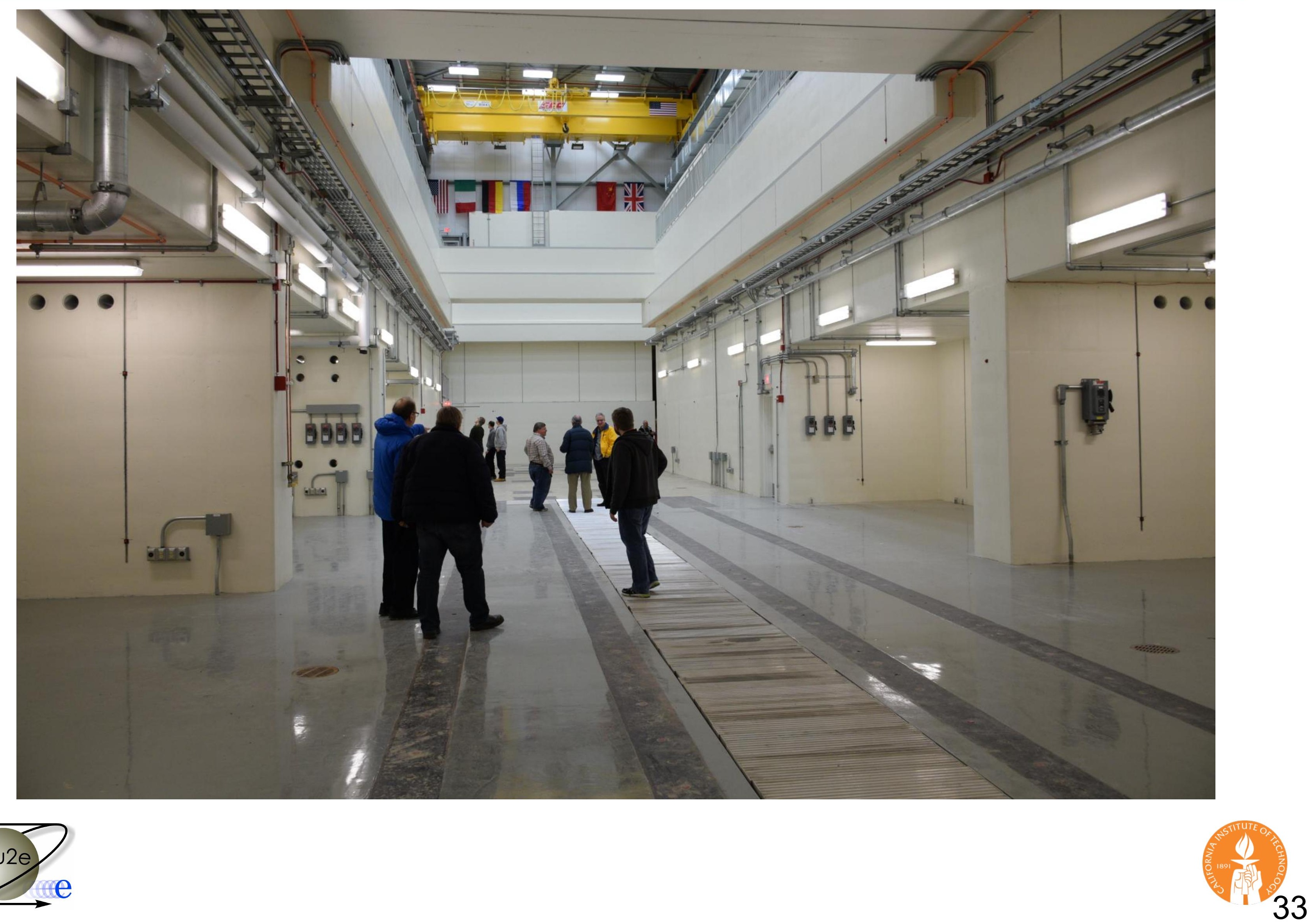




\section{Mu2e Schedule}

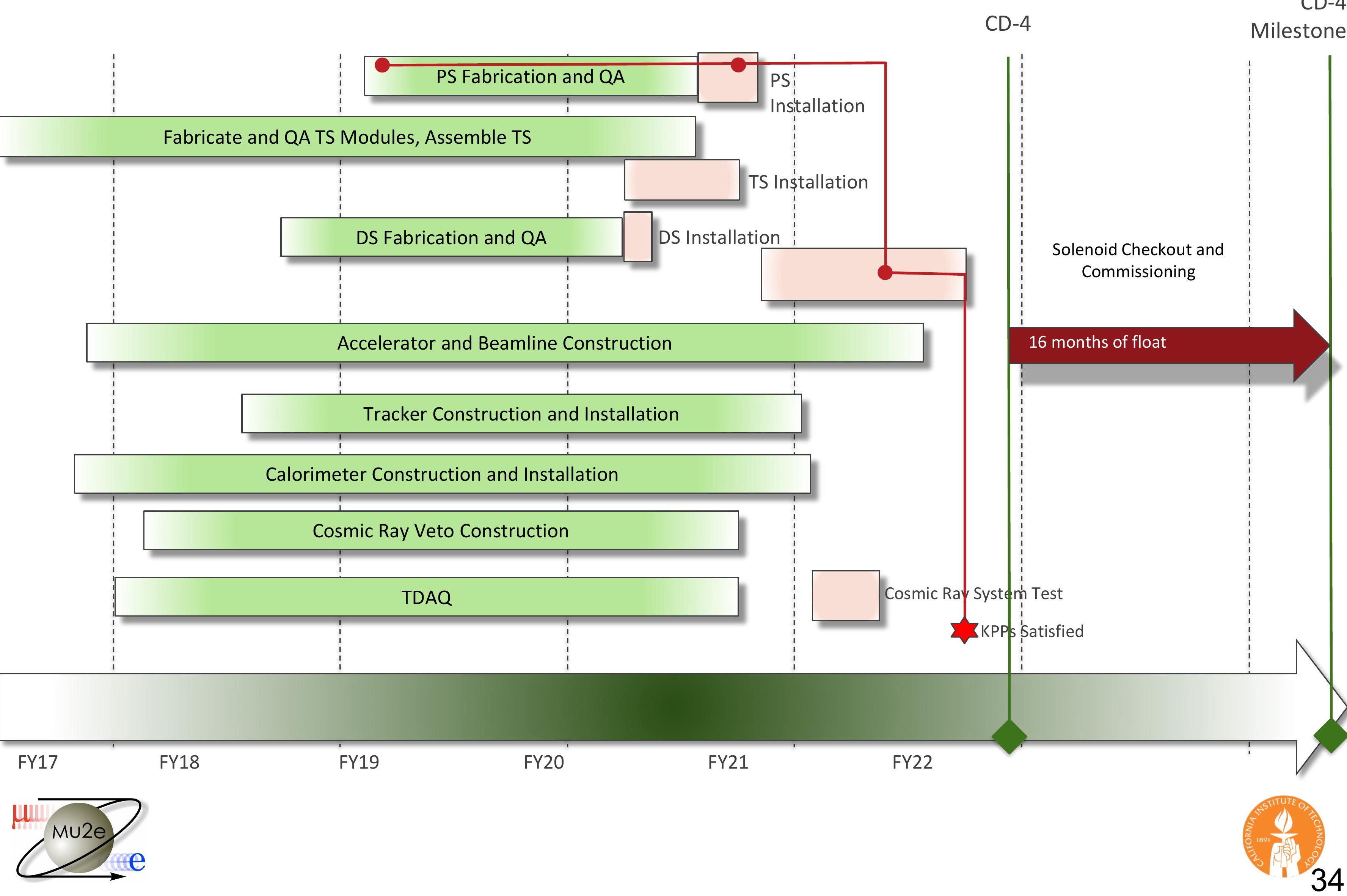




\section{Z-Dependence of $\mu \rightarrow e$ Conversion}

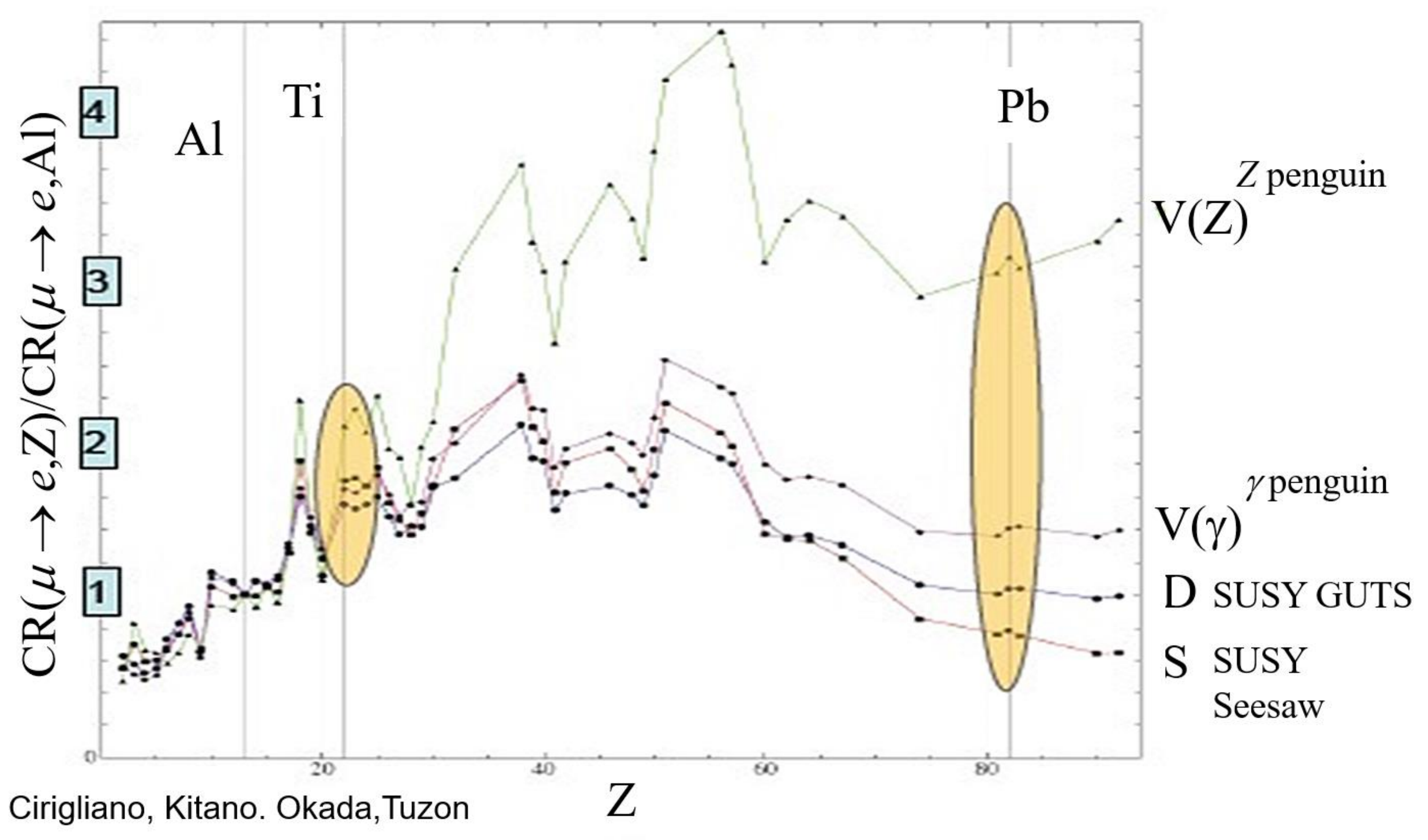




\section{Mu2e II Introduction}

- As Mu2e approaches commissioning, we are also looking toward future upgrades

- The proposed Mu2e II experiment aims to achieve an order of magnitude improvement in sensitivity over Mu2e

- If there is no signal at Mu2e: We could extend our sensitivity to find a signal or set new limits

- If Mu2e does see something: We can improve our statistical significance and use different target materials to narrow down the NP processes involved

- To achieve a 10X improvement, we need:

- An upgraded proton source (already approved)

- Other upgrades to parts of the detector

- We aim to reduce costs by reusing parts of mu2e wherever feasible 


\section{Mu2e II Plans}

- So far, various studies of Mu2e II backgrounds, sensitivity, and radiation damage have been performed

- A series of Mu2e II workshops has been held and the collaboration is involved in the Fermilab PIP-II planning process (a superconducting linac for LBNF and the muon campus)

- PIP-II will have an energy of $800 \mathrm{MeV}$ (Mu2e's proton source is $8 \mathrm{GeV}$ ) which is below the anti-nucleon production threshold and will result in less background

- An expression of interest was recently submitted to the Fermilab PAC

- Timecale:

- Mu2e is expected to run for 4 years of data-taking at full intensity

- Assuming 2-3 years from the end of $\mathrm{Mu} 2 \mathrm{e}$ to the start of Mu2e II, Mu2e II could begin taking data around 2030

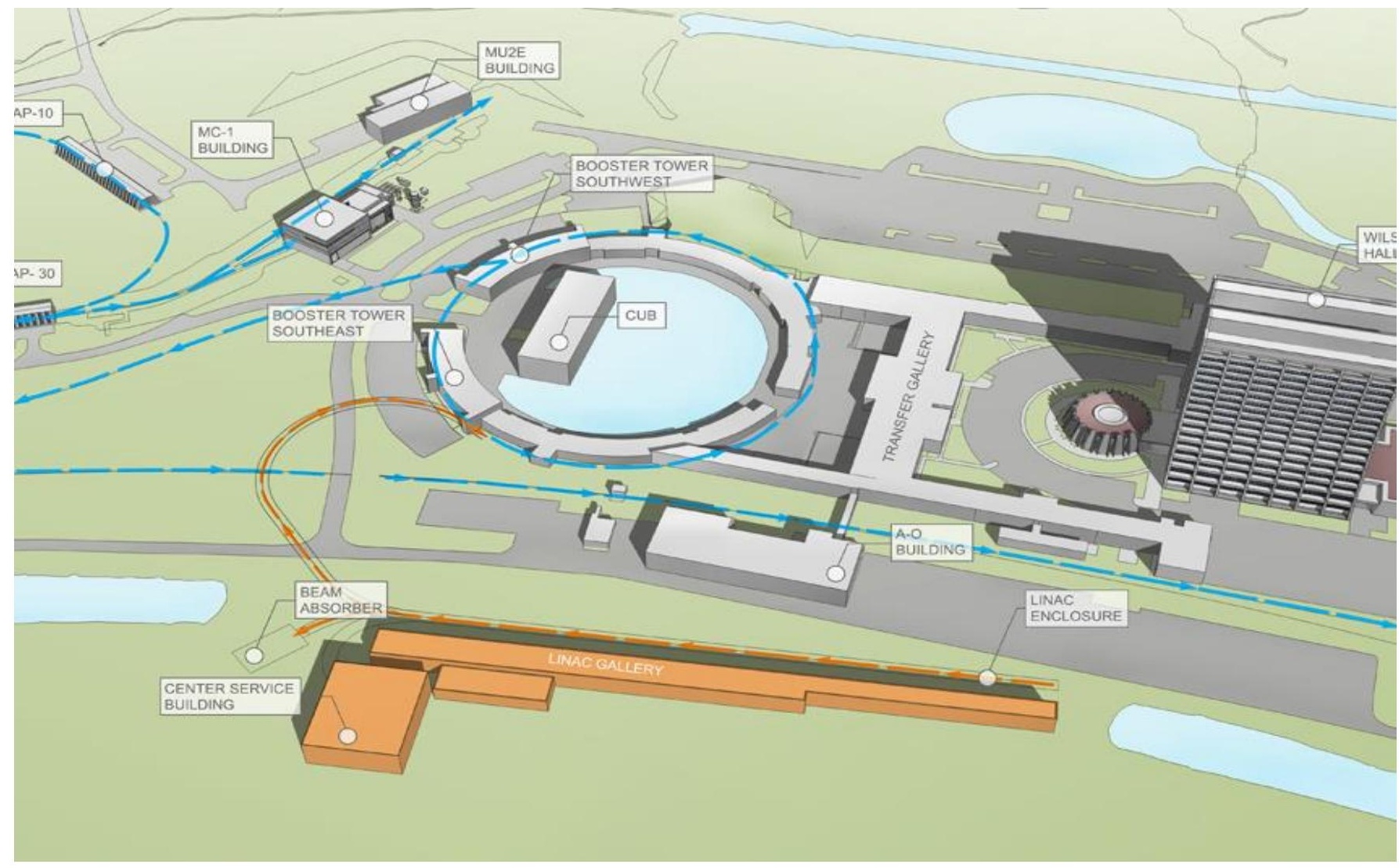




\section{Summary}

- The Mu2e experiment will improve current $\mu^{-} N \rightarrow e^{-} N$ CLFV sensitivity limits by 4 orders of magnitude (and thereby constrain many $\mathrm{NP}$ models at mass scales up to $\sim 10,000 \mathrm{TeV}$ )

- Mu2e will be sensitive to a broad range of NP models

- If we see a signal, switching to another stopping target material will provide further information about the Lorentz structure of the NP

- Progress is on schedule and we plan to begin commissioning in 2020 
Backup Slides 


\section{Purely Leptonic Conversion Processes}

$$
L_{\mathrm{CLFV}}=\frac{m_{m}}{(1+k) \Lambda^{2}} \bar{m}_{R} S_{m n} e_{L} F^{m n}+\frac{k}{(1+k) \Lambda^{2}} \bar{m}_{L} g_{m} e_{L}\left(\bar{e}_{L} g_{m} e_{L}\right)+\text { h.c. }
$$

$\Lambda$ : effective mass scale of New Physics $k$ : relative contribution of the contact term

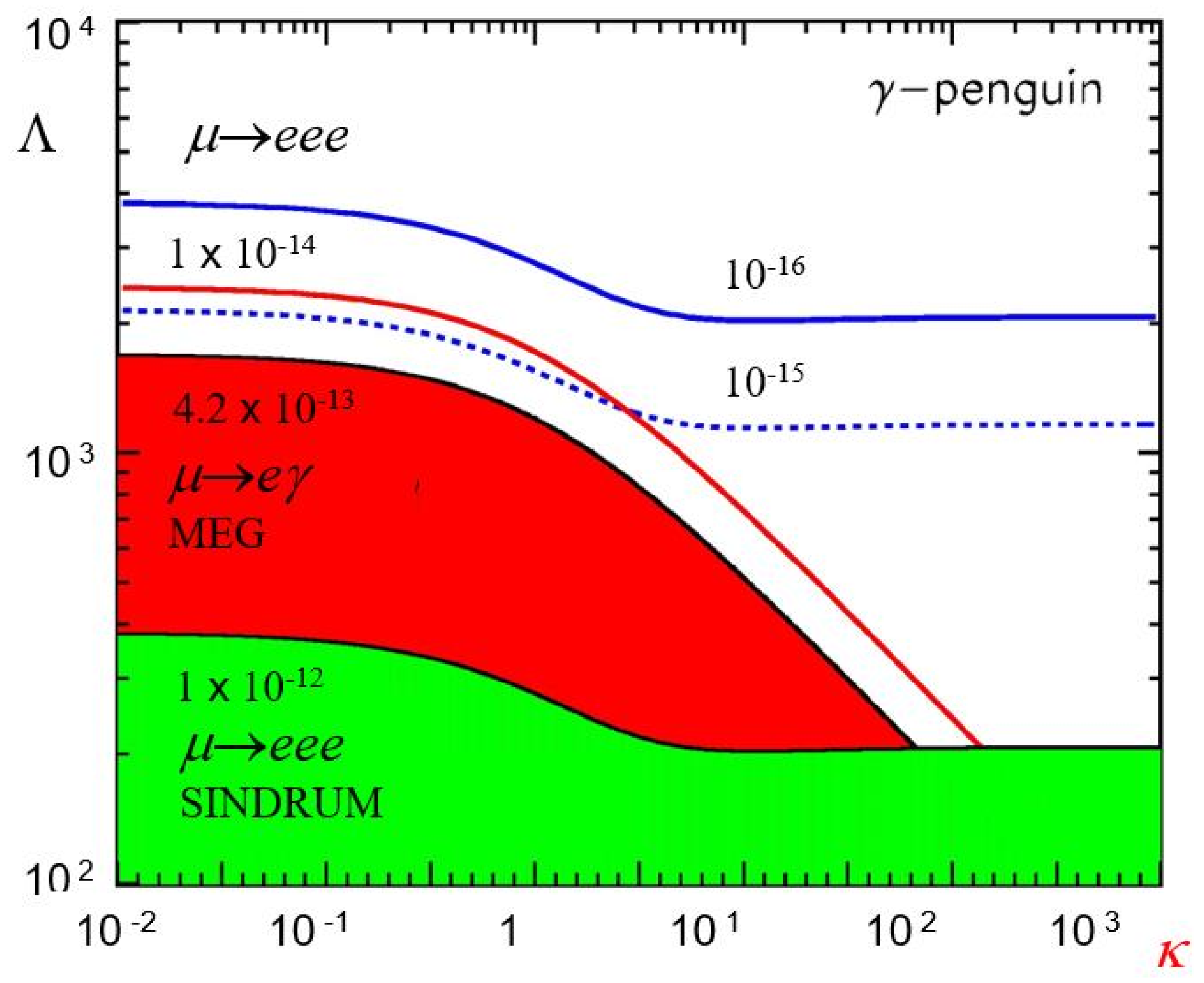

